\title{
The Phenylalanine Ammonia Lyase Gene LjPAL1 Is Involved in Plant Defense Responses to Pathogens and Plays Diverse Roles in Lotus japonicus-Rhizobium Symbioses
}

\author{
Yaping Chen, ${ }^{1,2}$ Fengjiao Li, ${ }^{1,2,3}$ Lu Tian, ${ }^{1,2,3}$ Mingchao Huang, ${ }^{1,2,3}$ Rufang Deng, ${ }^{1,2}$ Xueliu Li, ${ }^{1,2,3}$ \\ Wei Chen, ${ }^{1,2,3}$ Pingzhi Wu, ${ }^{1,2}$ Meiru Li, ${ }^{1,2}$ Huawu Jiang, ${ }^{1,2}$ and Guojiang Wu ${ }^{1,2, \dagger}$ \\ ${ }^{1}$ Key Laboratory of Plant Resources Conservation and Sustainable Utilization, South China Botanical Garden, Chinese \\ Academy of Sciences, Guangzhou 510650, China; ${ }^{2}$ Guangdong Provincial Key Laboratory of Applied Botany, South China \\ Botanical Garden; and ${ }^{3}$ University of Chinese Academy of Sciences, Beijing 100049, China
}

Accepted 7 June 2017.

\begin{abstract}
Phenylalanine ammonia lyase (PAL) is important in the biosynthesis of plant secondary metabolites that regulate growth responses. Although its function is well-established in various plants, the functional significance of $P A L$ genes in nodulation is poorly understood. Here, we demonstrate that the Lotus japonicus PAL (LjPAL1) gene is induced by Mesorhizobium loti infection and methyl-jasmonate (Me-JA) treatment in roots. LJPAL1 altered PAL activity, leading to changes in lignin contents and thicknesses of cell walls in roots and nodules of transgenic plants and, hence, to structural changes in roots and nodules. LjPAL1-knockdown plants (LjPAL1i) exhibited increased infection thread and nodule numbers and the induced upregulation of nodulin gene expression after $M$. loti infection. Conversely, LJPAL1 overexpression delayed the infection process and reduced infection thread and nodule numbers after $M$. loti inoculation. LjPALIi plants also exhibited reduced endogenous salicylic acid (SA) accumulation and expression of the SA-dependent marker gene. Their infection phenotype could be partially restored by exogenous SA or Me-JA application. Our data demonstrate that $L j P A L 1$ plays diverse roles in L. japonicus-rhizobium symbiosis, affecting rhizobial infection progress and nodule structure, likely by inducing lignin modification, regulating endogenous $\mathrm{SA}$ biosynthesis, and modulating SA signaling.
\end{abstract}

Plants continuously interact with both pathogenic and symbiotic soil microorganisms. Pathogenic microbes secrete effectors into host plant cells to suppress the host's immune response and, thereby, facilitate infection. This induces defense responses in the plant, such as the thickening of cells walls and the activation of the salicylic acid (SA) and jasmonic acid (JA) defense pathways to challenge the pathogen (Jones and Dangl 2006).

It has been reported that plant defense response pathways are also important in rhizobium-legume symbioses. The Arabidopsis

${ }^{\dagger}$ Corresponding author: G. Wu; E-mail: wugj@ scbg.ac.cn;

Telephone: +86.020.37252750; Fax: +86.020.37252703.

*The $\boldsymbol{e}$-Xtra logo stands for "electronic extra" and indicates that three supplementary figures and two supplementary tables are published online.

(c) 2017 The American Phytopathological Society thaliana gene NPRI (nonexpressor of pathogenesis-related gene1), which plays a key role in the SA-mediated defense pathway, was found to exhibit negative regulatory activity in symbiotic interactions, inhibiting root hair deformation and nodulin expression (Peleg-Grossman et al. 2009). Moreover, rhizobial nod factor induced FLS2 degradation and suppressed microbeassociated molecular pattern-triggered immunity in Arabidopsis (Liang et al. 2013). Studies on soybeans have shown that, in these legumes, the nucleotide binding site-leucine rich repeat-type disease-resistance genes $R j 2$ and $R f g 1$ are involved in the determination of symbiotic specificity (Yang et al. 2010). Finally, defense responses induced by the peptide flg22 inhibited rhizobial infection and delayed nodule organogenesis (Lopez-Gomez et al. 2012). Effective legume-rhizobium symbiosis requires at least partial suppression of the host plant's defense responses, but the mechanisms by which this suppression occurs are not well-known. To understand them better, it will be necessary to characterize the intersection between symbiosis and pathogenesis in model plants such as the legumes Medicago truncatula and Lotus japonicus.

In symbioses between legume plants and rhizobia, the host plant produces root nodules in response to Nod factors secreted by the microbes (Madsen et al. 2010). These Nod factor signals are essential for root-hair deformation, induction of early nodulation genes, rhizobial infection, and the formation of nodule primordia. At the cellular level in the model legumes L. japonicus and Medicago truncatula, infection is initiated by hydrolysis and invagination of plant cell plasma membranes, after which the bacteria are able to move down through the root-hair cells to form an infection thread (IT) (Geurts and Bisseling 2002). More generally, in mutualistic interactions such as root nodule symbioses and arbuscular mycorrhiza (AM), the cell walls of the plant host must permit the passage of the microbial partner to enable the establishment of the intimate interfaces required for developmental coordination and nutrient exchange (Rich et al. 2014).

Phenylpropanoid compounds are precursors to a wide range of phenolic compounds with many functions in plants (Dixon and Paiva 1995). Lignin, which is synthesized from phenylpropanoid compounds, is a major structural component of secondarily thickened cell walls (Boerjan et al. 2003). Phenylalanine ammonia lyase (PAL; EC 4.3.1.24) catalyzes the conversion of phenylalanine to transcinnamic acid, which is the first step in the phenylpropanoid pathway and an important point of regulation at the intersection of primary and secondary metabolism. In most 
plant species, the PAL proteins are encoded by a small family of genes with one to five members (Cochrane et al. 2004; de Jong et al. 2015; Huang et al. 2010; Raes et al. 2003; Reichert et al. 2009; Tsai et al. 2006), although members of genus Eucalyptus have 11 homologous PAL genes (Carocha et al. 2015) and watermelon has 12 (Dong and Shang 2013). Several studies have shown that $P A L$ gene expression responds to a variety of environmental stimuli, including pathogen infection, wounding, nutrient depletion, UV irradiation, extreme temperatures, and other stress conditions (Dixon and Paiva 1995; Payyavula et al. 2012). In Arabidopsis, which has four PAL genes (PAL1 to PAL4), pall and pal2 single mutants exhibited normal growth and development phenotypes but a pall/pal2/pal3/pal4 quadruple mutant exhibited greatly reduced levels of lignin, accumulated much lower levels of SA than the wild type, after pathogen infection, and was more susceptible to a virulent bacterial pathogen (Huang et al. 2010). Moreover, $P A L$-suppressed transgenic tobacco exhibited reduced SA levels in its leaves and undeveloped systemic acquired resistance (Pallas et al. 1996). This is consistent with the assumption that $C a P A L 1$ acts as a positive regulator of SA-dependent defense signaling to combat microbial pathogens via its enzymatic activity in the phenylpropanoid pathway (Kim and Hwang 2014). Similarly, in the monocot plant genus Brachypodium, PAL knockdown resulted in reduced lignin accumulation and increased susceptibility to fungal pathogens.

In the roots of $L$. japonicus, the expression of two PAL genes (MWM056d02_r/Lj1g3v4590850 and GENLf025c04/Lj1g3v459076 0.2 ) was significantly up-regulated 2 days after Mesorhizobium loti inoculation, but their expression fell dramatically upon prolonged inoculation (Kouchi et al. 2004). Deguchi et al. (2007) reported that the $L$. japonicus genome contains ten $P A L$ genes. Of these, the transcript levels of four $L j P A L$ genes-AB283031 (Lj1g3v4590840.1), AB283032/AB283038 (Lj1g3v4590850.2), AB283033 (Lj1g3v4590840.1), and AB283034/AB283035 (Lj1g3v4590760) — in the roots decreased after 6 weeks of inoculation with AM fungi and 4 weeks after inoculation with M. loti. However, the effects of the $P A L$ genes on nodulation in L. japonicus and the mechanisms by which these effects occur remain unclear. To bridge this knowledge gap, we describe here the identification and characterization of the LjPAL1/Lj1g3v4590760.2 gene from L. japonicus. Having identified this gene, LjPAL1 knockdown (LjPALIi) and overexpression plants $(L j P A L 1-O E)$ were used to further investigate the function of $L j P A L 1$ in nodulation. The results obtained demonstrate that PAL plays multiple roles in controlling both root architecture and symbiotic processes in L. japonicus.

\section{RESULTS}

\section{Identification and expression analyses of $P A L$ genes in $L$. japonicus.}

Searches of $L$. japonicus whole-genome sequences (The Lotus japonicus genome browser) using Arabidopsis PAL homologs as queries revealed ten distinct $P A L$ genes, which were designated LjPAL1 to LjPAL10 (Supplementary Table S1). To clarify the evolutionary relationships of these genes, a phylogenetic tree was constructed, based on the sequences of the LjPAL proteins and publicly available sequence data for PAL proteins from four monocots and five dicots, using the neighbor-joining method as implemented in the Mega4 program (Supplementary Fig. S1). This analysis placed the PALs of the dicots and monocots into separate groups, as previously reported by Wu et al. (2014). Although all of the $L j P A L s$ clustered into the dicotyledon group, they were subdivided across four distinct classes. $L j P A L 1$, LjPAL2, LjPAL3, LjPAL4, and LjPAL5 are tandem repeats located on chromosome I and belong to the same class. LjPALA and LjPAL5 (Lj1g3v4590850.1 and Lj1g3v4590850.2) are two separate genes, and both of them contain a complete open reading frame (ORF), even though they have the same accession number and appear as splicing variants of the same locus.

To investigate the expression patterns of the $P A L$ genes in $L$. japonicus, we examined their transcript levels in leaves, stems, roots, pods, and nodules. Transcripts of $L j P A L 1, L j P A L 2$, LjPAL3, LjPAL4, LjPAL5, LjPAL7, and LjPAL8 were detected in all tested tissues. However, there was no detectable expression of $L j P A L 10$ in stems and no detectable expression of LjPAL6 and LjPAL9 in any tested tissue. Furthermore, compared with the expression level of other $L j P A L$ genes, $L j P A L 1$ and $L j P A L 2$ showed high expression in both roots and nodules (Fig. 1A). Next, the expression of $L j P A L$ genes in roots after exogenous SA and JA treatment was analyzed by real time polymerase chain reaction (PCR). The expression of $L j P A L 1$, $L j P A L 2, L j P A L 3$, and $L j P A L 10$ in roots could be dramatically up-regulated by methyl-jasmonate (Me-JA) treatment (Fig. $1 \mathrm{~B})$, but there was no significant change in the expression of $L j P A L$ genes in roots following treatment with exogenous SA (Fig. 1C). Consequently, no data for the expression of $L j P A L$ genes other than LjPAL1 and LjPAL2 are shown.

To understand the function of $P A L$ genes in rhizobiumlegume symbioses, we investigated the expression patterns of $\angle j P A L$ genes at different time points after $M$. loti inoculation. The transcript levels of $L j P A L 6$ and $L j P A L 9$ were undetectable in both uninoculated and inoculated roots. In addition, the expression of LjPAL3, LjPALA, LjPAL5, LjPAL7, LjPAL8, and LjPAL10 genes had no significant change in roots, following inoculation with $M$. loti. Nevertheless, the expression of LjPAL1 and LjPAL2 increased significantly on the first day of root infection with $M$. loti but, then, decreased after 3 days (Fig. 1D). This suggests that LjPAL1 and LjPAL2 are involved in nodulation. Because of its strong expression in both nodules and uninoculated roots and the pronounced increase in its expression induced by $M$. loti infection, the LjPALI gene was selected for further investigation.

\section{LJPAL1 is expressed mainly in roots and nodules.}

To further investigate the expression of LjPALI in L. japonicus, we generated transgenic plants harboring a fusion of the putative $L j P A L 1$ promoter and $\beta$-glucuronidase (GUS). GUS signals were observed in the roots, nodule primordia, and mature nodules (Fig. 2A, C, and D). About $80 \%$ of the roots and nodules evaluated had GUS signals as that shown in Figure 2A and D. The image in Figure 2C is representative of 23 of 30 nodule primordia evaluated. Analyses of transverse sections of roots and nodules showed that $L j P A L 1$ was mainly expressed in vascular bundles of roots and mature nodules (Fig. 2B, E, and $\mathrm{F})$ and also in the cortex tissues of nodule primordia and mature nodules (Fig. 2C, E, and G).

\section{LjPAL1i and LjPAL1-OE transgenic plants exhibit altered PAL activity and lignin levels.}

To clarify the function of LjPALI in L. japonicus, 18 transgenic L. japonicus MG-20 lines were prepared-12 independent $L j P A L 1$ RNA interference lines ( $L j P A L 1 i)$ and six independent LjPAL1-overexpressing lines (LjPAL1-OE). Homozygous descendants of the transgenic lines were then chosen for further analysis. The discussion below is based on studies of three LjPAL1-OE lines (OE-1, OE-3, and OE-4) and three LjPAL1i lines (i-1, i-9, and i-12).

The expression of $L j P A L 1$ genes in 2-week-old roots was analyzed using quantitative reverse transcription (RT)-PCR, revealing that the abundance of $L j P A L 1$ transcripts in the roots of the LjPALIi lines (i-1 and i-12) was much lower than in the wild type; conversely, the transcript was much more abundant in the LjPAL1-OE lines (Fig. 3A). To determine whether any 

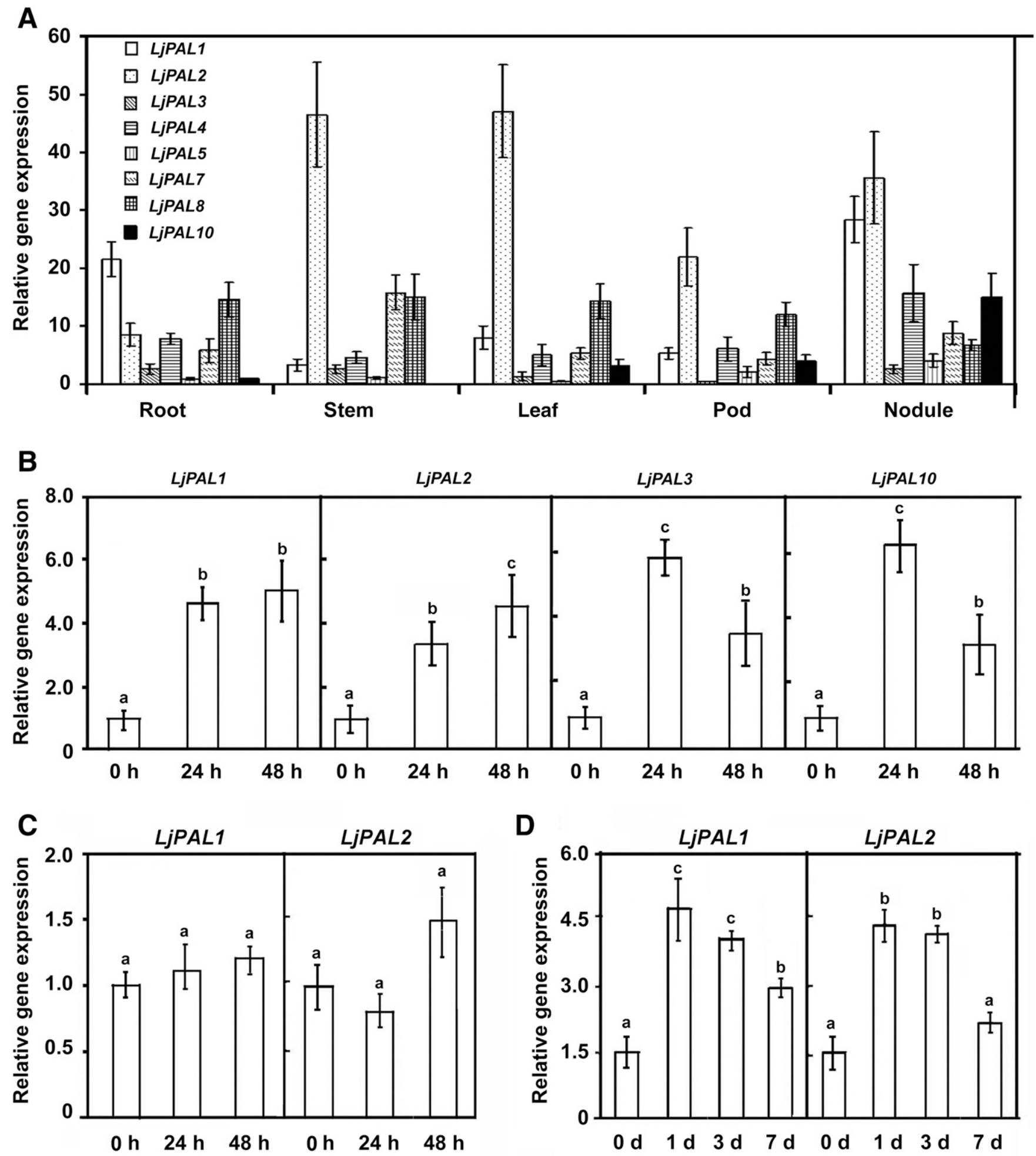

Fig. 1. Expression patterns of $L j P A L$ genes in Lotus japonicus MG-20. A, Tissue-specific expression patterns of $L j P A L$ genes in roots, stems, leaves, pods, and nodules. Transcripts of $L j P A L 6$ and $L j P A L 9$ could not be detected in the tested tissues. B, Expression of $L j P A L 1$ and its homologous genes in roots following treatment with $100 \mu \mathrm{M}$ methyl-jasmonate (Me-JA). $L j P A L 1, L j P A L 2, L j P A L 3$, and $L j P A L 10$ were up-regulated in response to Me-JA, but the expression of other $L j P A L$ genes was either undetectable in roots or there was no change in response to the treatment. C, Expression of $L j P A L 1$ and its homologous genes in roots following treatment with $100 \mu \mathrm{M}$ exogenous salicylic acid (SA). The expression of $L j P A L 1$ and $L j P A L 2$ was readily detected in both treated and untreated roots, but some of the $L j P A L$ genes were not induced by exogenous SA treatment. D, Expression of $L j P A L 1$ and its homologous genes in roots in response to infection by Mesorhizobium loti. Only the expression of $L j P A L 1$ and $L j P A L 2$ in roots was significantly induced by M. loti; the other $L j P A L$ genes were only weakly expressed or not detectable in uninoculated and inoculated roots. The data are averages of triplicate RNA preparations, and error bars indicate the standard errors of the data resulting from three independent experiments. Statistical significances according to Fisher's protected least significant difference test $(P<$ 0.05 ) are indicated by different letters above the data points. 
genes homologous to $L j P A L 1$ were affected by the RNA interference in the $L j P A L I i$ plants, the expression of other $L j P A L$ genes was also examined. The transcript level of $L j P A L 2$ was slightly reduced in LjPAL1i roots (Supplementary Fig. S2), indicating that the interfering RNA suppressed the expression of LjPALI and also (to a much lesser extent) one of its homologs.

To determine whether changes in PAL activity reflected changes in the abundance of the LjPALl transcript in the transgenic plants, crude protein extracts were prepared from 2 -week-old roots, and then, the conversion of L-phenylalanine to transcinnamate was assayed by tracking UV light absorbance increases attributable to transcinnamic acid accumulation. Comparing these measurements with a transcinnamic acid standard curve, it was determined that extracts from $L j P A L 1 i$ roots had, on average, 25\% lower PAL activity levels than the wild type, whereas extracts from $L j P A L 1-O E$ roots exhibited 90 to $150 \%$ greater PAL activity (Fig. 3B).

It is well-known that PAL is the first enzyme of the phenylpropanoid pathway, which generates precursors to many unique and important plant metabolites, such as lignins, suberins, and various phenolic esters (Wanner et al. 1995). Therefore, we investigated the total lignin contents of roots and nodules of the transgenic lines. On average, the lignin contents of the roots and nodules of the LjPALIi plants were 11 and $17.5 \%$ lower, respectively, than those of wild-type plants (Fig. 3C and D). More significantly, the OE-1 and OE-4 lines both had substantially greater amounts of lignin in their roots (37 and $46 \%$, respectively) and nodules (33 and 45\%, respectively) compared with the wild type (Fig. 3C and D). These results are consistent with the conclusion that changes in PAL activity yield corresponding changes in lignin content (Cass et al. 2015).
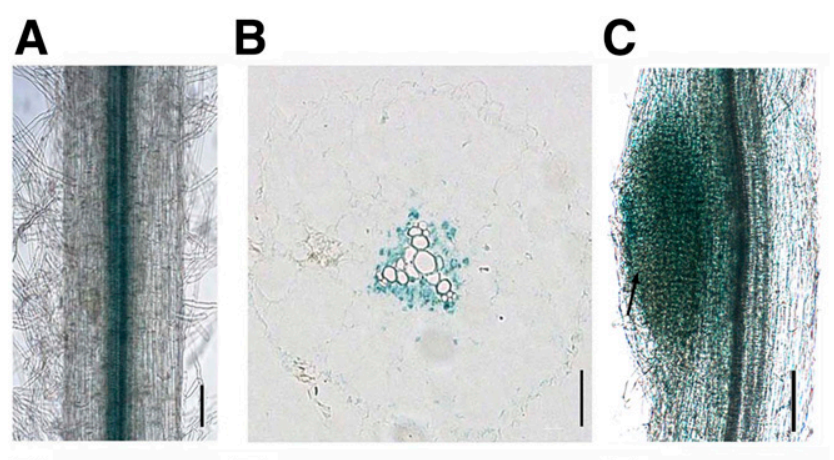

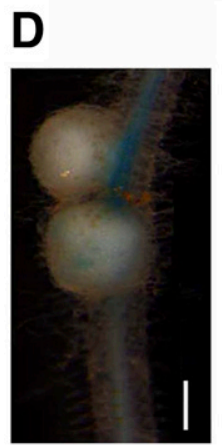

\begin{abstract}
E
\end{abstract}

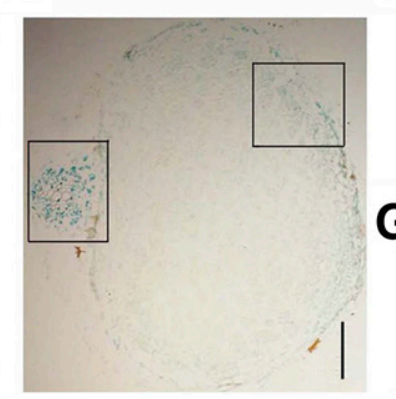

$\mathbf{F}$

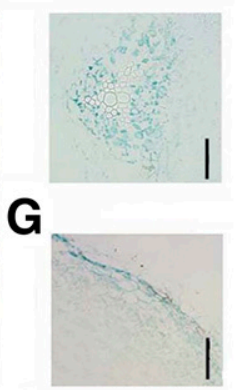

Fig. 2. Histochemical analysis of the expression of $\operatorname{Pro}_{L j P A L 1}$ :GUS in Lotus japonicus. A, Roots from 2-week-old seedlings. B, Transverse sections of 2 -week-old roots. C, Nodule primordium. Primordia were collected 3 days after Mesorhizobium loti inoculation. D, Mature nodules collected after roots had been inoculated with $M$. loti for 3 weeks. E, Transverse sections of 3-week-old nodules. F, Expansion of the region enclosed in the box on the left in E. G, Expansion of the region enclosed in the box on the right in E. For A, C, and D, $n=30$ to 40 and for B and E, $n=15$ to 20. Scale bars = $50 \mu \mathrm{m}$ in $\mathrm{B}, \mathrm{F}$, and $\mathrm{G}, 100 \mu \mathrm{m}$ in $\mathrm{A}, \mathrm{C}$, and $\mathrm{E}$, and $250 \mu \mathrm{m}$ in $\mathrm{D}$.
However, LjPAL1i line i-9 appears to have relatively poor silencing, and therefore, both PAL activity and root and nodule lignin from i-9 plants had no significant change.

\section{LjPAL1 transgenic plants exhibited modified root architecture.}

Because PAL is known to affect development in certain plants (Cass et al. 2015; Rohde et al. 2004), the developmental phenotype of the LjPAL1 transgenic plants was investigated. The developmental phenotype of the LjPALIi plants did not differ significantly from the wild type, but the LjPALI-OE plants had shorter and thicker roots (Fig. 4A and B). Transverse sections in the elongation zone of 2-week-old roots showed that the parenchymal cells in the roots of the LjPALI-OE plants were bigger than those in the wild type (Fig. 4B and D). The cell-wall thickness in these lines was further investigated by transmission electron microscopy (TEM), revealing that the cell walls in the OE-1 roots were 50\% thicker than in the wild type (Fig. 4C and E). This demonstrates that $L j P A L 1$ affects root development and influences cell wall modification in L. japonicus.

\section{LjPAL1 affects rhizobial infection and nodule structure.}

To clarify the effect of LjPAL1 on nodulation in L. japonicus, the nodulation phenotype of $L j P A L 1 i$ and $L j P A L 1-O E$ plants was analyzed in detail. Compared with the wild type, the number of ITs increased in LjPALIi plants and decreased in LjPAL1-OE plants at both 7 days and 2 weeks after their roots were inoculated with $M$. loti (Fig. 5B). The number of nodules in the LjPALI i and LjPAL1-OE lines changed accordingly at 2 and 4 weeks after inoculation (Fig. 5A and C). However, the nodule numbers in 6-week-old roots of the transgenic lines did not differ substantially from those observed in the wild type (Fig. 5C). In addition, compared with the wild type, the LjPAL1 $i$ lines exhibited smaller nodules at 4 weeks after M. loti infection (Fig. 5D), which was consistent with the finding that reduced lignin levels caused the formation of small nodules in a shikimate hydroxycinnamoyl transferase (HCT)-downregulated alfalfa (Gallego-Giraldo et al. 2014).

The microstructure of 3-week-old nodules was analyzed to clarify the function of LjPAL1 in nodule development. (Fig. 6). A light micrograph of transverse sections of nodules did not show obvious difference between the wild-type and transgenic plants (Fig. 6A). The TEM results showed that the sclereid cells surrounding the nodules in the LjPALI-OE lines were larger than those in the wild type (Fig. 6B and D), and the cell walls in the LjPAL1-OE nodules were also thicker than those in wildtype nodules (Fig. 6C and D). However, in both the LjPALI $i$ and LjPAL1-OE lines, the morphology of bacterioids in the nodules was identical to those observed for the wild type (Fig. 6C). Acetylene reduction assays performed at various timepoints after infection revealed no significant differences in nitrogenase activity between wild-type and LjPALI $i$ nodules. While 15- and 30-day-old LjPAL1-OE nodules exhibited lower nitrogenase activity than the wild type, no such difference was observed for 45-day-old nodules (Fig. 6E).

Taken together, these results suggest that $L j P A L 1$ influences the progress of rhizobial infection and nodule organogenesis during the early stages of rhizobium-L. japonicus symbiosis and also affects nodule development by modifying the structure of the nodules but not their nitrogenase activity (Figs. 5 and 6).

\section{The transgenic plants exhibit modified expression of nodulin genes.}

Nodulin gene expression is a distinct indicator of symbiotic signal perception. Early nodulin genes (ENOD) are the earliest induced genes and are used as molecular markers for rhizobial infection (Takeda et al. 2005). Moreover, it has been suggested 
that the NODULE INCEPTION (NIN) gene is required for both rhizobial infection and nodule organogenesis (Yoro et al. 2014). Both rhizobial infection and nodule organogenesis were altered in $L j P A L 1 i$ and $L j P A L 1-O E$ lines, so we investigated the effect of $L j P A L 1$ on nodulin gene expression. Compared with the wild type, the transcripts of the nodulin genes LjENOD4O and LjNIN were more abundant in infected roots of $L j P A L 1 i$ plants and less abundant in those of the LjPAL1-OE lines (Fig. 7). These results indicate that $L j P A L 1$ may act as a regulator of rhizobial infection in rhizobium-legume symbiosis.

SA accumulation and SA-dependent defense gene expression are altered in $L j P A L 1$ transgenic plants after $M$. loti inoculation.

PAL plays an important role in plant defense against pathogens because of its function in SA biosynthesis (Chaman et al. 2003; Mauch-Mani and Slusarenko 1996; Nugroho et al. 2002). The bacterial pathogens Pseudomonas syringae pv. tomato DC3000 and P. syringae pv. phaseolicola NPS3121(NPS3121) were used to infect L. japonicus MG-20, revealing that MG-20 is susceptible to NPS3121 (Fig. 8A). LjPAL1 transgenic plants were then infected with NPS3121. By day 7 after inoculations, larger areas of necrotic lesions in i-1 plants were visible on roots than those in MG-20, but in contrast, roots of OE-4 plants were virtually free of lesions (Fig. 8B). Compared with MG-20, LjPAL1i plants exhibited increased susceptibility to NPS3121, while the LjPAL1-OE line exhibited enhanced resistance to NPS3121 (Fig. 8B). The expression of SA-dependent marker genes in these plants was investigated by real-time RT-PCR, revealing that, upon infection with NPS3121, the transcription of $L j P R 1 b$ decreased in $L j P A L 1 i$ plants relative to the wild type but increased in $L j P A L 1 i$-OE plants (Fig. 8C). Conversely, the levels of the JA-dependent marker gene $L j P D F 1$ were similar in wildtype and transgenic roots (Fig. 8D). Therefore, $L j P A L 1$ may act as a positive regulator of SA-dependent defense signaling that is induced by exposure to bacterial pathogens in L. japonicus.

Although the molecular basis of SA effects on nodulation is currently unclear, it is believed to affect legume-rhizobium interactions (Chen et al. 2014; Stacey et al. 2006). Because LjPAL1 induced SA-dependent defense responses in L. japonicus
A
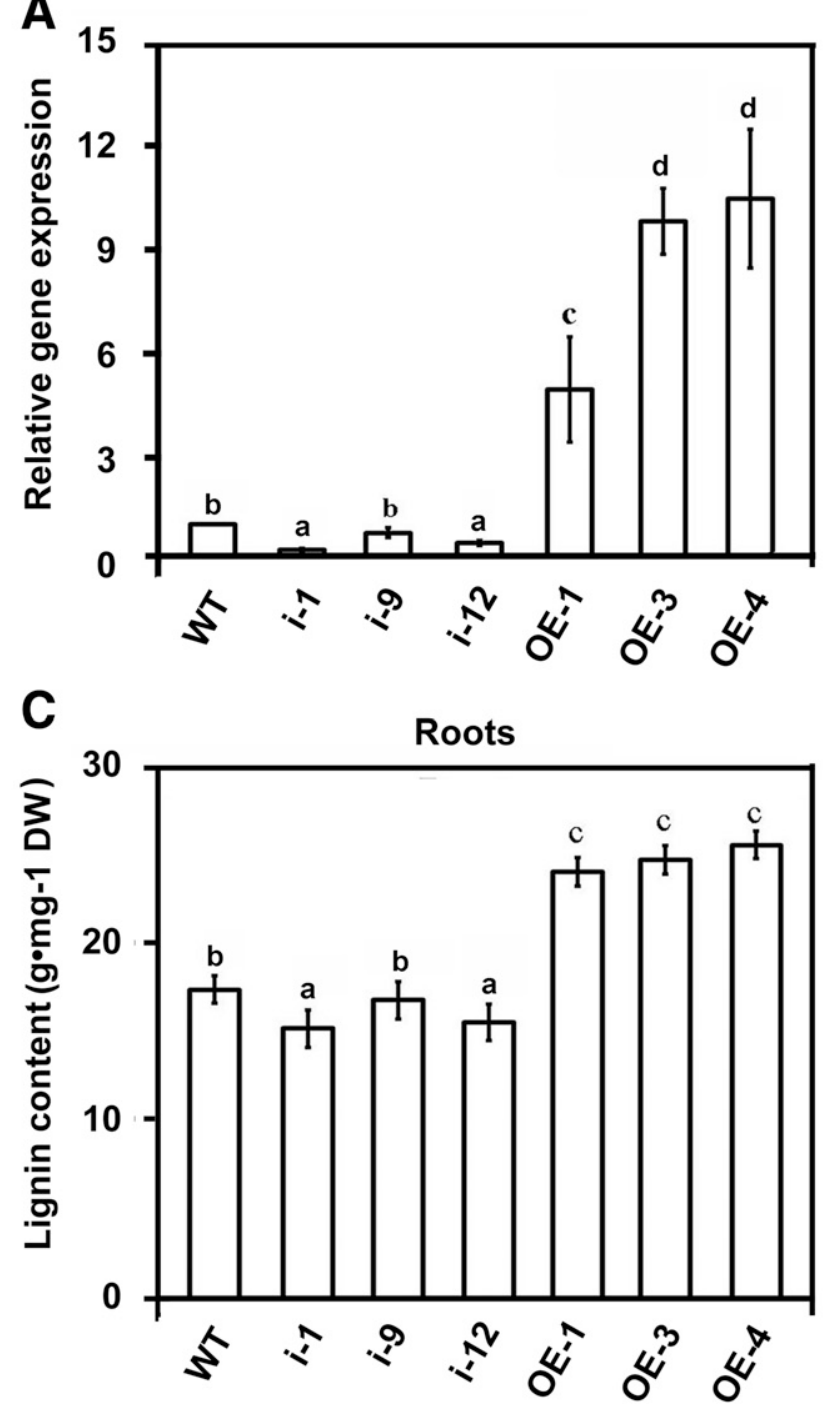

B

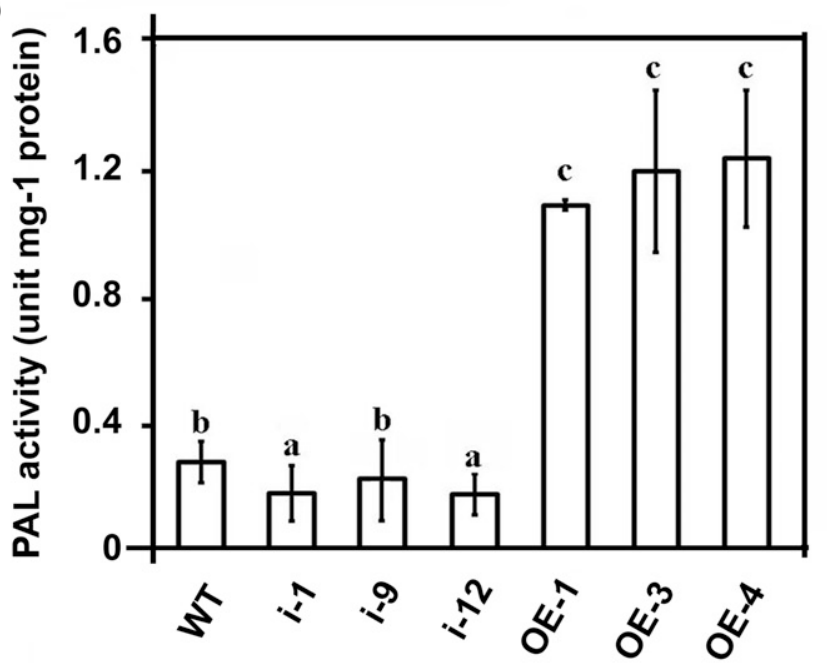

D

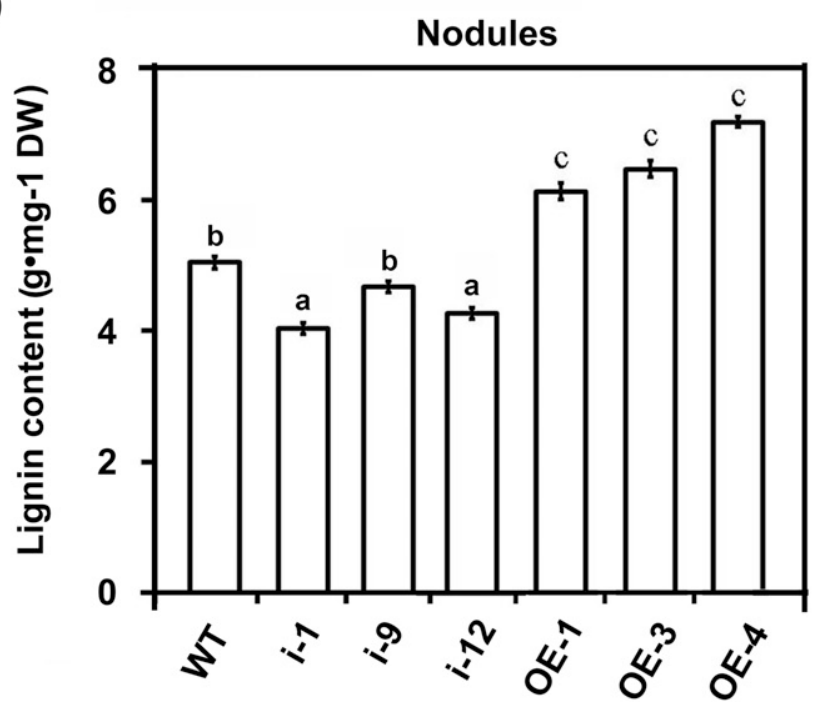

Fig. 3. Changes in phenylalanine ammonia lyase (PAL) activity and lignin contents in $L j P A L 1$ transgenic plants. A, Real-time polymerase chain reaction analysis of the transcript levels of $L j P A L 1$ in roots of wild type, $L j P A L 1 i$, and $L j P A L 1$-OE (overexpressing) lines. The data are averages of triplicate RNA preparations, and error bars indicate the standard errors of the data resulting from three independent experiments. B, Detection of PAL activity in wild-type and $L j P A L 1$ transgenic roots. Measurements of PAL activity were performed in triplicate. C, Measurement of lignin contents in roots of wild-type and transgenic plants. Roots were collected when plants were 2 weeks old. D, Measurements of lignin content in nodules of wild-type and transgenic plants. Nodules were collected after roots had been inoculated with Mexorhizobium loti for 3 weeks. Data in B, C, and D represents four biological replicates and three technical replicates. Statistical significances according to Fisher's protected least significant difference test $(P<0.05)$ are indicated by different letters above the data points. 
following exposure to a bacterial pathogen (Fig. 8), we investigated the accumulation of SA and the expression of related genes in the roots of this species following inoculation with $M$. loti. To this end, total SA levels (i.e., free
SA plus glucose-conjugated SA) in the roots of the wildtype and $L j P A L 1$ transgenic lines were measured during $M$. loti infection. The LjPAL1-OE lines exhibited significantly higher total SA levels than the wild type, whereas the
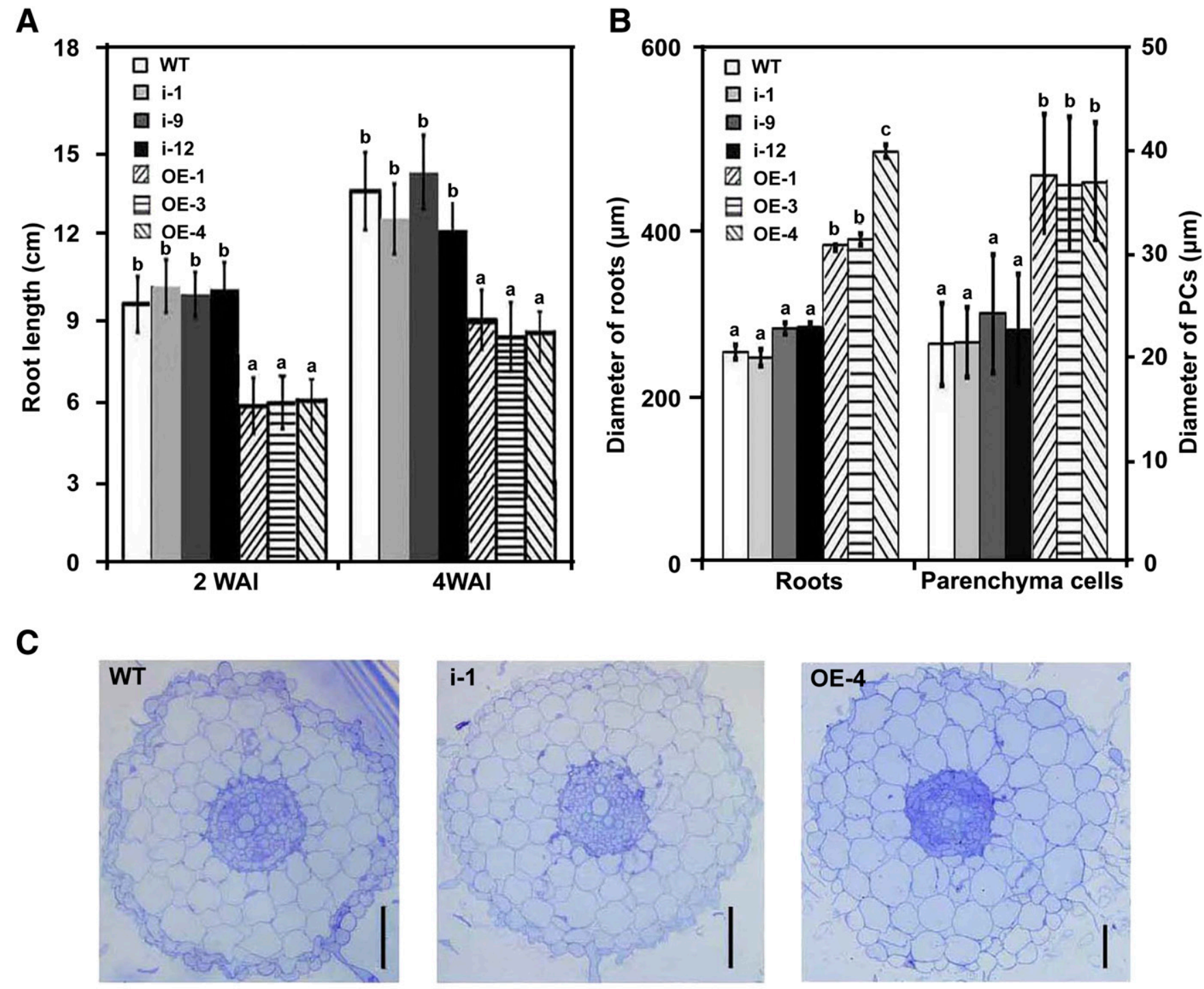

D

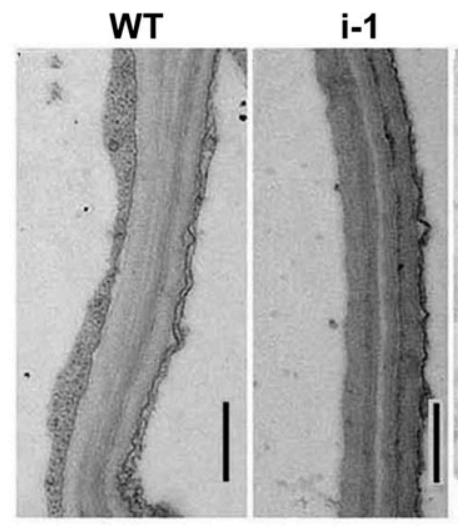

E

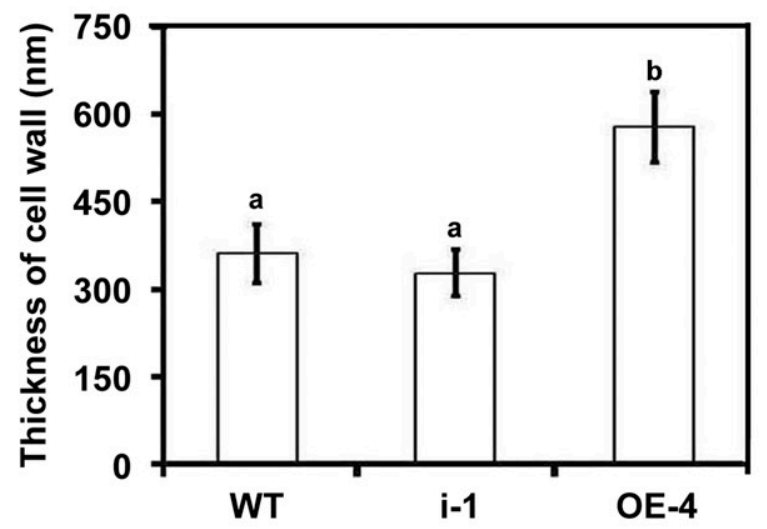

Fig. 4. Root development phenotypes of $L j P A L 1$ transgenic plants. A, Root length at 2 and 4 weeks after germination. B, Diameter measurement of 2-week-old roots and diameter of parenchyma cells. C, Transverse sections of 2-week-old roots. D, Transmission electron microscopy images of parenchymal cell walls in 2 -week-old roots. E, Thickness of the cell wall in parenchyma cells; $n=40$ for root lengths and 20 for parenchyma cells. Statistical significances according to Fisher's protected least significant difference test $(P<0.05)$ are indicated by different letters above the data points. 
LjPAL1i lines exhibited rather lower levels (Fig. 9A). Subsequent real-time RT-PCR analyses showed that levels of $L j P R 10$ were significantly reduced in $L j P A L 1 i$ plants but substantially increased in $L j P A L 1-O E$ lines during $M$. loti infection (Fig. 9B). In contrast, the levels of $L j P D F 1$ were similar in wild-type and $L j P A L 1$ transgenic roots (Fig. 9C). Together with the observed nodulation phenotypes of the transgenic plants (Fig. 5), these results suggest that $L j P A L 1$ acts as a regulator of SA-dependent defense signaling following rhizobial infection in L. japonicus.

The rhizobial infection phenotype of $L j P A L 1 i$ lines is partially restored by exogenous SA or Me-JA application.

Changes in LjPAL1 expression affected endogenous SA levels (Fig. 9A). To determine whether SA has strong effects on nodulation in the LjPAL1i lines, the roots of plants from these lines were treated with exogenous SA for $6 \mathrm{~h}$ and, then, were inoculated with $M$. loti; $50 \mu \mathrm{M}$ SA did not inhibit the growth of M. loti (Supplementary Fig. S3) and, thus, was chosen for the treatment. Treatment with SA did not change the number of ITs in the wild-type plants but dramatically reduced the number of ITs in all the transgenic plants 7 days after inoculation with M. loti (Fig. 10A and B). Therefore, exogenous SA treatment partially restored the rhizoidal infection phenotype of $L j P A L 1 i$ plants, suggesting that endogenous SA and its defense signaling should be a component of the function of $L j P A L 1$ in L. japonicus nodulation.

Previous studies have revealed that the plant hormone JA can activate the phenylpropanoid pathway (Pauwels et al. 2008; Taheri and Tarighi 2010). Because $10 \mu \mathrm{M}$ exogenous JA had no
A

\section{MG-20 i-1 i-9 i-12 OE-1 OE-3 OE-4}

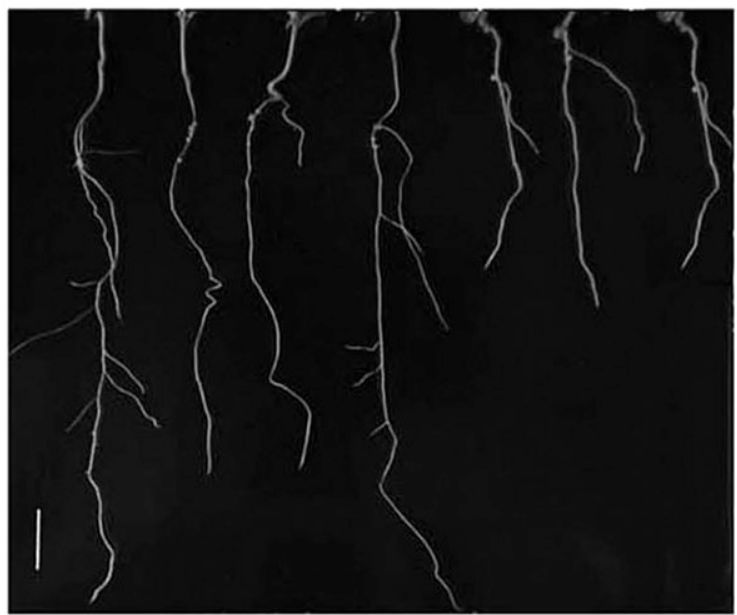

C

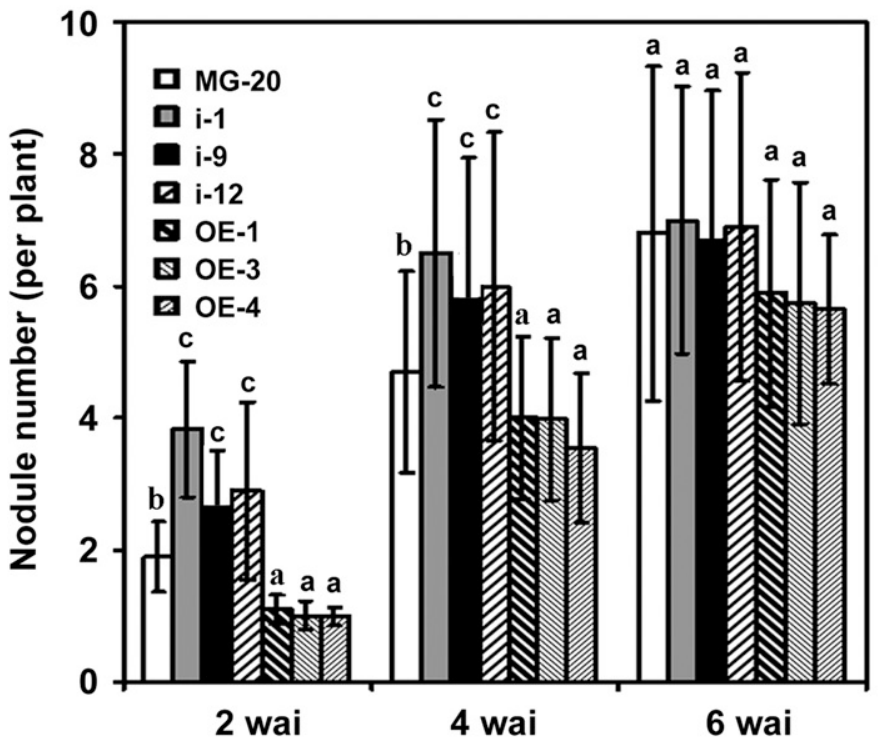

B

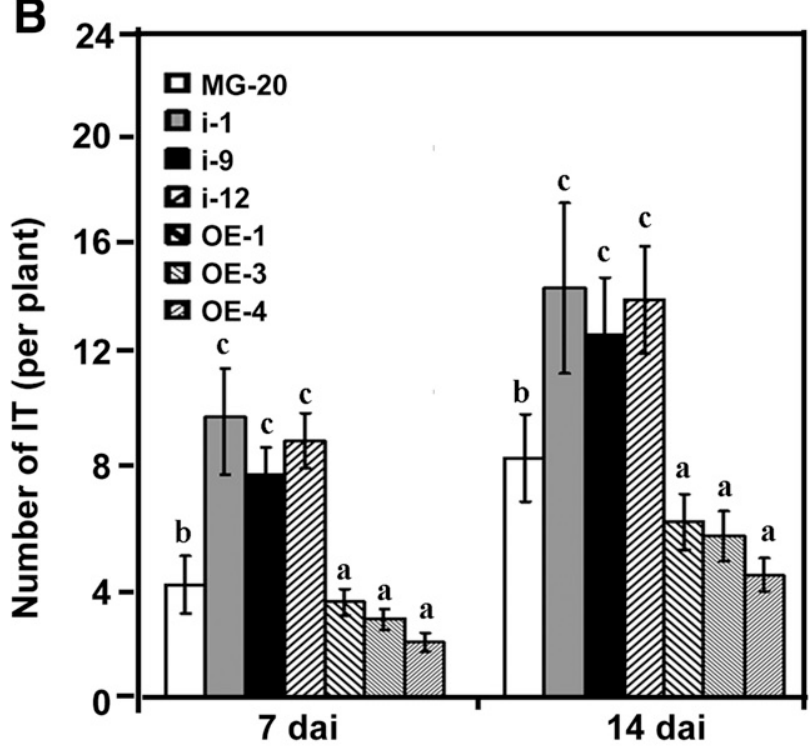

D

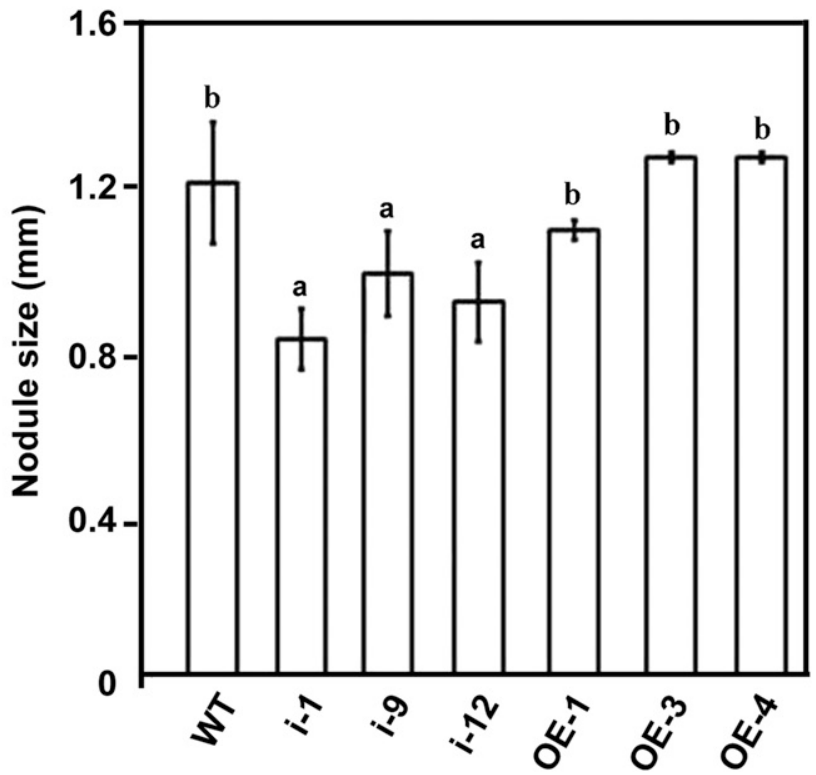

Fig. 5. Symbiotic phenotypes of $L j P A L 1$ transgenic plants. A, Illustrative photographs of MG-20 and $L j P A L 1$ transgenic plants grown in magenta boxes and inoculated with Mesorhizobium loti for 2 weeks. B, Number of infection threads (ITs) formed per root 7 and 14 days after inoculation with M. loti expressing DsRED for MG-20 and $L j P A L 1$ transgenic lines grown on agar slants supplemented with 1 mM $\mathrm{KNO}_{3}$. C, Number of nodules per plant 2, 4, and 6 weeks after inoculation with $M$. loti. D, Sizes of the largest root nodules in wild-type (WT) plants and various transgenic lines 3 weeks after inoculation with $M$. loti. Each experiment was performed in triplicate; $n=40$ to 50 for ITs, nodule counts, and nodule size measurements. Statistical significances according to Fisher's protected least significant difference test $(P<0.05)$ are indicated by different letters above the data points. 
A

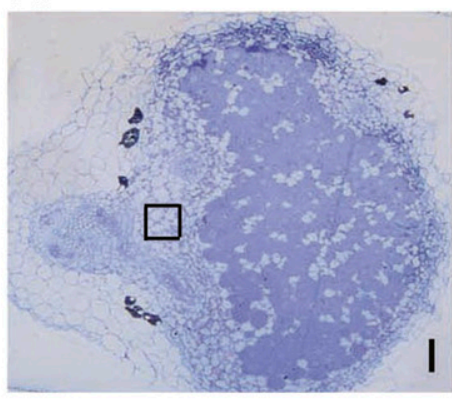

B

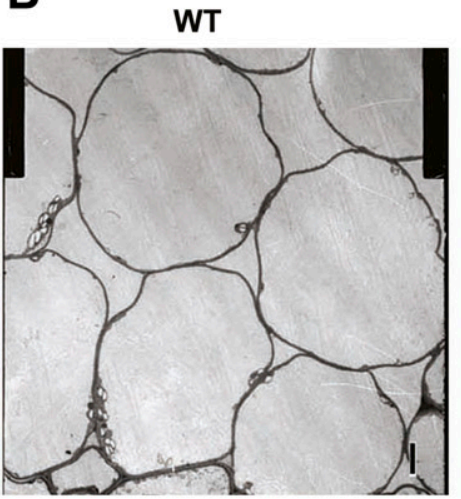

C

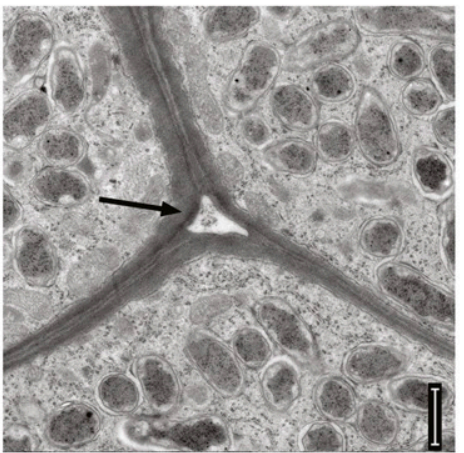

D

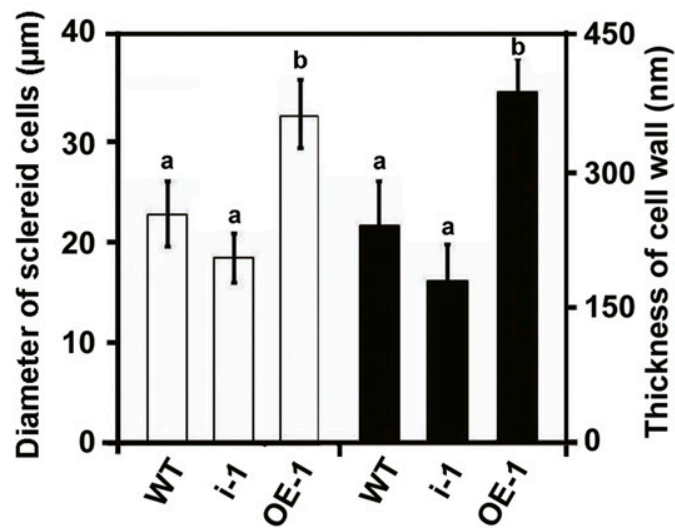

i-1

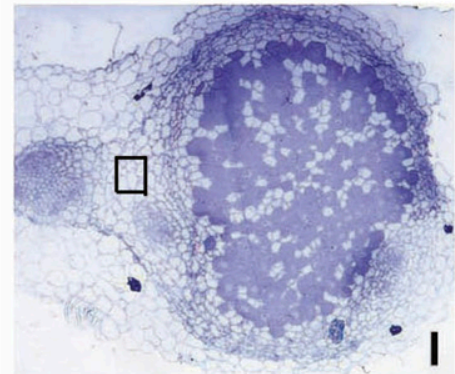

i-1

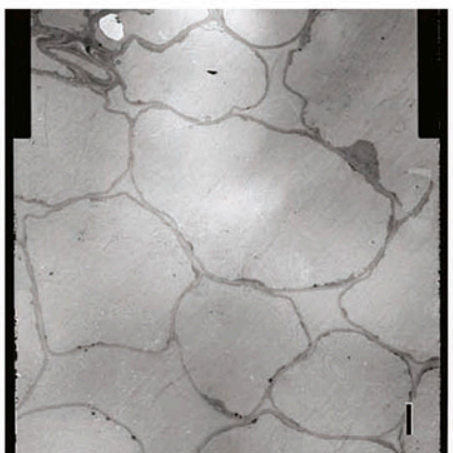

i-1

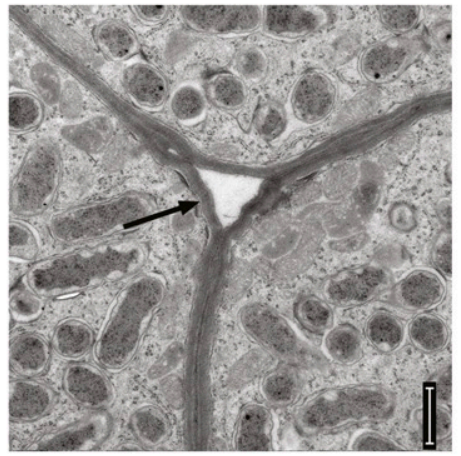

OE-1

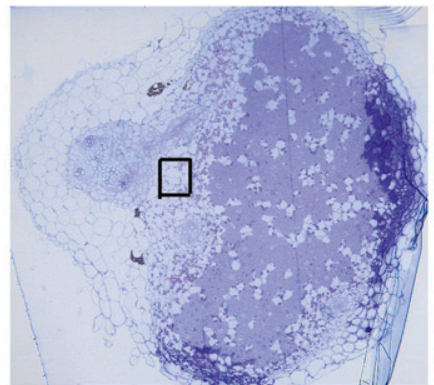

OE-1

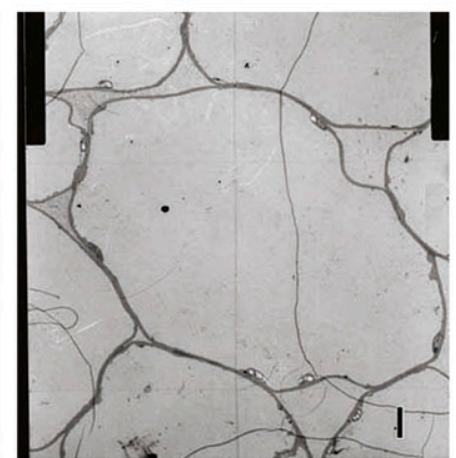

OE-1

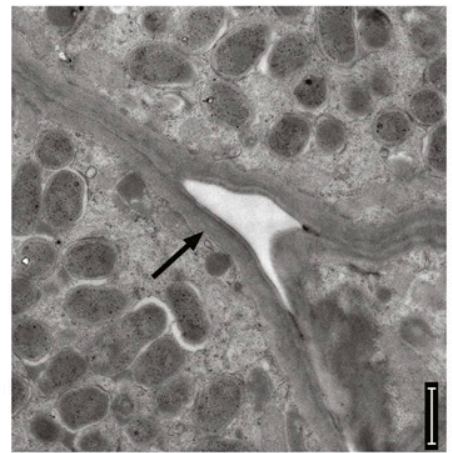

E

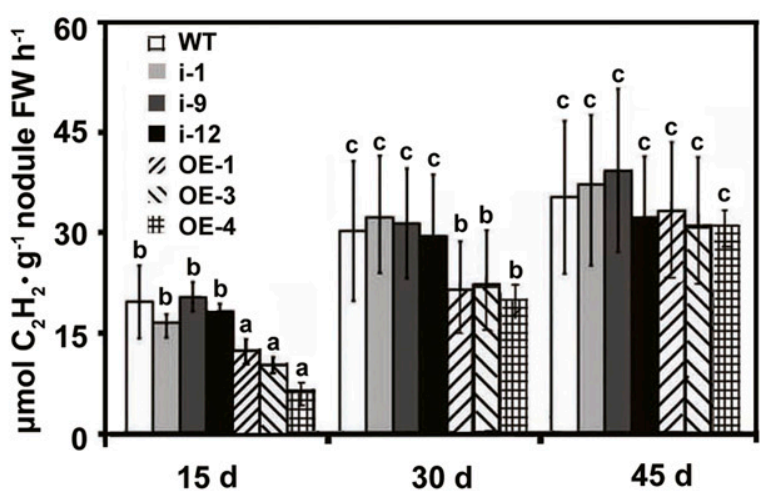

Fig. 6. Characterization of nodules on wild type and transgenic plants. A, Nodule sections of MG-20, $L j P A L 1$ RNA interference line 1 (i-1), and $L j P A L 1$ overexpressing line 1 (OE-1) inoculated with Mesorhizobium loti for 3 weeks. B, Transmission electron microscopy (TEM) images of sclereid cells surrounding 3-week-old nodules from MG-20, i-1, and OE-1 roots, close-up images of the boxed areas the in A. C, TEM images of the infected zone in 3-weekold nodules on MG-20, i-1, and OE-1 roots. Arrows indicate the cell wall measured in D. D, Measured diameters of sclereid cells in B and thicknesses of cell wall in C. E, Nitrogenase activity per gram of fresh weight (FW) of 15-, 30-, and 45-day-old nodules, as measured by the acetylene reduction assay; $n=15$ to 20 nodules for section in each experiment. Statistical significances according to Fisher's protected least significant difference test $(P<0.05)$ are indicated by different letters above the data points. Bar $=5 \mu \mathrm{m}$ in $\mathrm{A}, 500 \mathrm{~nm}$ in $\mathrm{B}$, and $1 \mu \mathrm{m}$ in $\mathrm{C}$. 
apparent effect on the growth of $M$. loti, Me-JA at this concentration was chosen for application on plants.

In the absence of Me-JA treatment, there was a clear and substantial difference between the wild-type plants and the $L j P A L 1 i$ line with respect to the number of ITs formed upon M. loti infection. This difference was abolished by Me-JA treatment to some extent (Fig. 10A). The proportion of the reduced ITs in LjPALI $i$ and LjPALI-OE lines is much larger than that in the wild-type plants (Fig. 10B). It thus appears that the nodulation phenotype of LjPAL1i plants could be partially restored by the treatment with Me-JA. Together with the finding that the expression of $L j P D F 1$ was not changed in $L j P A L 1 i$ plants (Figs. 8D and 9B), this result indicates that JA can activate the phenylpropanoid pathway to regulate nodulation in L. japonicus. To clarify the effect of exogenous JA on the nodulation of $L j P A L l i$ plants, the expression of $L j P A L$ genes in roots was investigated by real time RT-PCR. The $L j P A L 1$ homologous genes $L j P A L 2, L j P A L 3, L j P A L 6, L j P A L 8$, and $L j P A L 10$ were weakly expressed in roots when plants were grown without environmental stress (Fig. 1A). However, with the exception of $L j P A L 1$, the expression of these homologous genes in $L j P A L 1 i$ roots was up-regulated dramatically upon treatment with Me-JA (Fig. 10C). The restoration of the nodulation phenotype in the LjPALIi lines following Me-JA treatment can thus be attributed to the induction of the expression of PAL1 homologs, which compensate for the suppression of LjPAL1 expression during the early stages of legume-rhizobium symbiosis.

\section{DISCUSSION}

The biological functions of the $P A L$ genes have previously been studied by silencing them in some plants and by generating mutants in Arabidopsis (Cass et al. 2015; Huang et al. 2010; Kim and Hwang 2014; Pallas et al. 1996; Rohde et al. 2004). Here, we investigated their functions in legume-rhizobium symbiosis by studying $L j P A L 1$-silenced (LjPAL1i) and LjPAL1overexpressing ( $L j P A L 1-O E)$ plants. Our analysis of these transgenic plants has provided novel information about the different roles of $P A L$ genes in responses to environmental elements in legume plants.
The levels of $P A L$ gene expression in different tissues of $L$. japonicus vary in response

to plant hormone treatments and $M$. loti inoculation.

Some members of the PAL gene family in L. japonicus have previously been identified by Deguchi et al. (2007). However, inspection of the miyakogusa.jp 3.0 database suggests that some of the PAL genes identified by these authors are duplicates. The ten $P A L$ genes identified from whole-genome sequences (L. japonicus genome assembly build 3.0) in this work were named according to their chromosomal position in the L. japonicus genome.

It has been shown that the PAL gene families in various species are expressed differentially in different plant tissues and in response to stress (Dixon and Paiva 1995; MacDonald and D'Cunha 2007). In Arabidopsis, PAL1, PAL2, and PALA are strongly expressed in inflorescent stems, whereas the PAL3 transcript is expressed at a very low level (Raes et al. 2003). Similar results were obtained for $L$. japonicus, whose ten $P A L$ genes were transcribed at very different levels in the studied tissues (Fig. 1A). This observation is consistent with the finding that willow $P A L$ genes have different expression levels in roots, phloem, xylem, stem tissue, and leaves (de Jong et al. 2015). A previous report showed that the willow genome features a tandem duplication comprised of the genes SVPAL2-1 and $S v P A L 2-2$, both of which appear to have retained their functionality (de Jong et al. 2015). Similarly, the L. japonicus genome includes five tandem gene duplicates ( $L j P A L 1, L j P A L 2$, $L j P A L 3, L j P A L 4$, and $L j P A L 5$ ) that cluster in the same clade and appear to have retained their functionality but have different expression patterns (Fig. 1). The LjPAL genes could thus play independent roles in legume development. This hypothesis is supported by the finding that treatment with Me-JA and exposure to $M$. loti both affect the expression of the individual $L j P A L$ genes in different ways (Fig. $1 \mathrm{~B}$ and D). Exogenous SA application induced the expression of $P A L$ genes in tobacco, tomato, parsley, Salvia officinalis, and Salvia virgate, and a positive correlation was observed between the concentration of exogenous SA and PAL expression in shoots (Ejtahed et al. 2015). Our results showed that the expression of ten $P A L$ genes in L. japonicus roots did not change significantly following SA treatment (Fig. 1C). However, it will be necessary to conduct
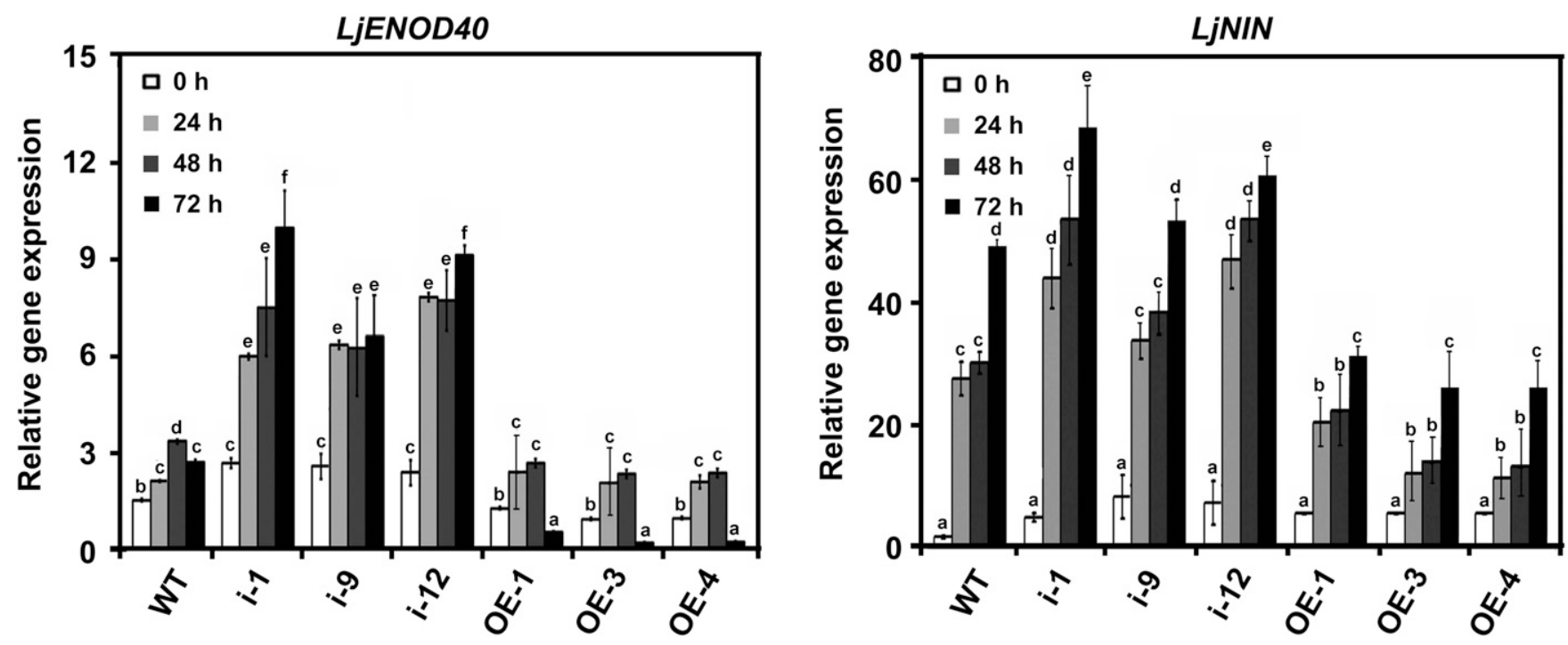

Fig. 7. Real-time polymerase chain reaction analysis of the expression of early nodulin genes in roots inoculated with Mesorhizobium loti at different time points. The nodule inception gene $(L j N I N)$ and early nodulin gene (LjENOD4O) were analyzed. The data are averages of triplicate RNA preparations, and error bars indicate the standard errors of the data resulting from three independent experiments. Statistical significances according to Fisher's protected least significant difference test $(P<0.05)$ are indicated by different letters above the data points. 
experiments with a wider range of exogenous SA concentrations to fully clarify the relationship between SA levels and the expression of the $L j P A L$ genes. Moreover, plant cells activate several defense mechanisms upon perception of JA signals, resulting in a massive reprogramming of gene expression. In Arabidopsis, Me-JA elicitation of cell cultures significantly induced expression of the AtPAL2 gene, which is consistent with the upregulation of $L j P A L$ genes in the roots of $L$. japonicus upon treatment with Me-JA (Fig. 1B).

Many defense-response genes were up-regulated during the early stages of Medicago truncatula-rhizobium symbiosis but were subsequently downregulated (Lohar et al. 2006). In keeping with their role as defense response genes, the expression of the $P A L$ genes was up-regulated during the early stages of both rhizobial and AM fungal infection in L. japonicus and was downregulated in the later stages (Deguchi et al. 2007; Kouchi et al. 2004) (Fig. 1D). Therefore, defense signaling mediated by $P A L$ genes appears to be rapidly activated but subsequently suppressed in legume-rhizobium interactions, which is likely to facilitate IT progression into the root cortex (Lohar et al. 2006).

\section{LjPAL1 affects root architecture and the progress of rhizobial infection in $L$. japonicus, likely via modification of lignin.}

Knocking down PAL genes in tobacco and Brachypodium distachyon reduced lignin levels and delayed development in plants (Cass et al. 2015; Elkind et al. 1990). Similar results were observed in Arabidopsis pal1/pal2 double mutants, which exhibited reduced PAL activity along with decreased lignin accumulation and altered cell-wall ultrastructure (Rohde et al. 2004). These results were also confirmed in our study (Figs. 3 , 4 , and 6). Interestingly, while both pal1/pal2 double mutants and pal1/pal2/pal3/pal4 quadruple mutants exhibit reduced lignin levels and abnormal growth development (Huang et al. 2010; Rohde et al. 2004), the knockdown of LjPAL1 in
A
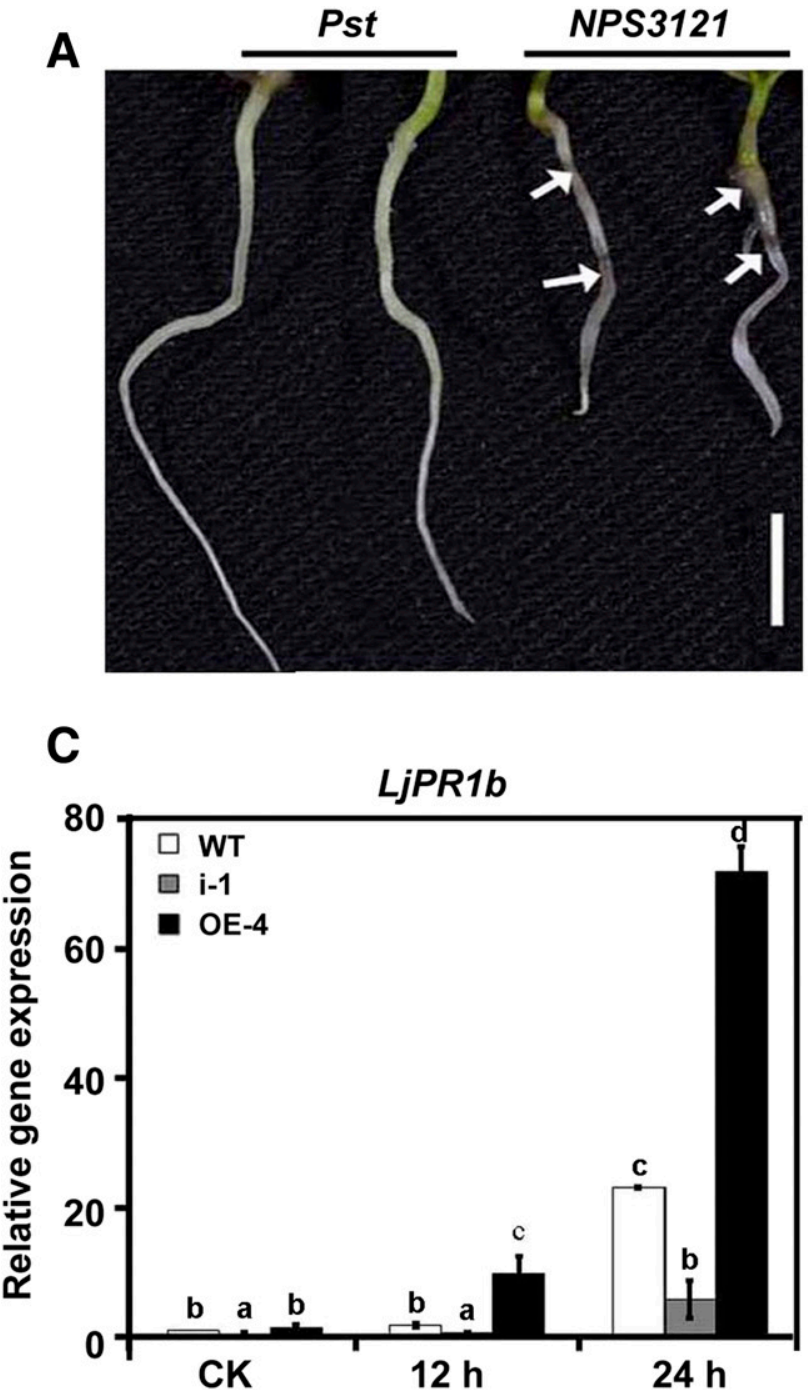

B

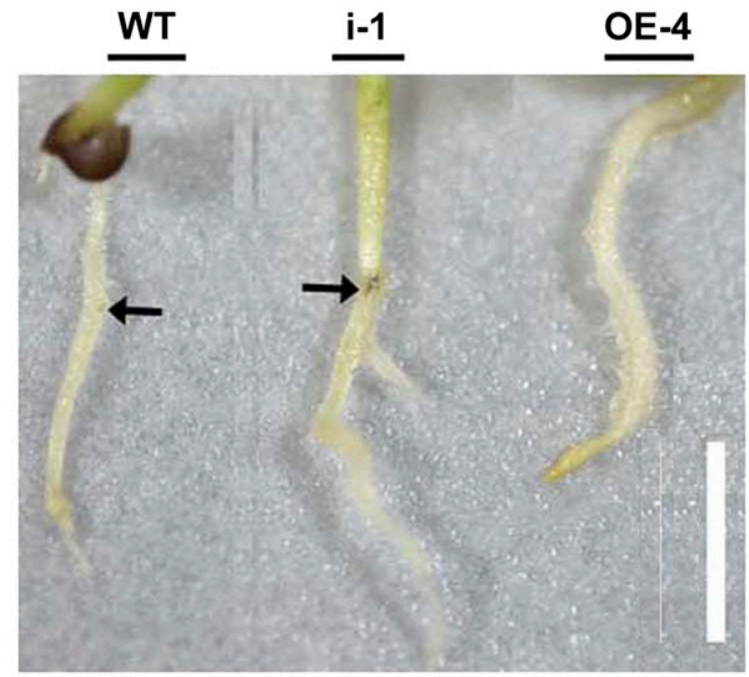

D

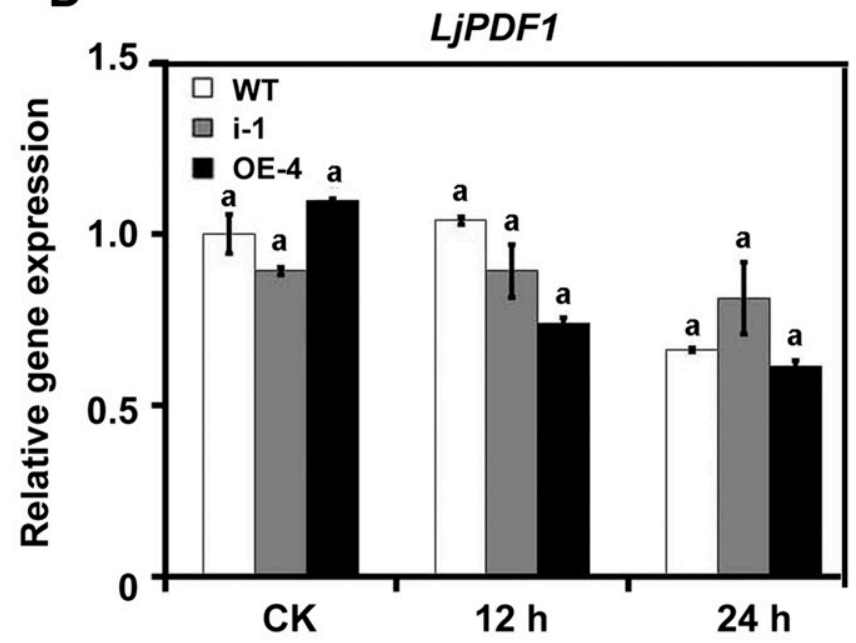

Fig. 8. Susceptibility of $L j P A L 1$ RNA interference ( i-1) and $L j P A L 1$ overexpressing (OE-1) transgenic plants to infection by bacterial pathogen. A, Disease symptoms developed on wild type (WT) after inoculation with bacterial pathogen Pseudomonas syringae pv. tomato DC3000 (Pst) and P. syringae pv. phaseolicola NPS3121 (NPS3121), respectively, $n=40$ plants for inoculation, and plant assay experiments were repeated at least three times with similar results. B, Disease symptoms developed on wild type, i-1, and OE-1 after inoculation with $P$. syringae pv. phaseolicola NPS3121, $n=20$ plants for inoculation in each experiment, and plant assay experiments were repeated at least three times with similar results. C and D, Expression of salicylic acid-dependent marker gene $L j P R 1 b$ and jasmonic acid-dependent marker gene $L j P D F 1$ in wild-type and transgenic roots was checked after NPS3121 treatment at different time points, as indicated. The data are averages of triplicate RNA preparations and error bars indicate the standard errors of the data resulting from three independent experiments. Statistical significances according to Fisher's protected least significant difference test $(P<0.05)$ are indicated by different letters above the data points. 
L. japonicus did not have any clear phenotypic consequences in the shoots. This is probably because $L j P A L 1$ was primarily expressed in the roots and nodules (Figs. 1 and 2).

Perturbations in lignin biosynthesis affect secondary cellwall composition in plants. Lignin confers rigidity to cell walls and, therefore, influences plant tolerance of biotic and abiotic stresses as well as their mechanical stability (Frei 2013). Previous studies have prompted the suggestion that accumulations of phenylpropanoid compounds, particularly lignin, may form a mechanical barrier to microbial infection in some plants (Cass et al. 2015; Frei 2013; Huang et al. 2010). In keeping with this hypothesis, in the legume Medicago sativa, reduced lignin levels are associated with increased nodule numbers (GallegoGiraldo et al. 2014). In L. japonicus, the changes in lignin levels seen in $L j P A L 1$ transgenic plants affected the thickness of root cell walls (Figs. 3, 4, and 6) and, thus, modified the mechanical barriers to rhizhobial infection. This led directly to changes in the number of ITs and nodules. These results may explain why the $L j P A L 1 i$ plants exhibited increased numbers of nodules whereas the LjPAL1-OE plants had fewer nodules than the wild type (Figs. 5 and 6). Changes in the effectiveness of the mechanical barriers presented by lignin either accelerated or delayed nodule organogenesis in $L j P A L 1$ transgenic plants. However, whether lignin has a direct effect on the expression of nodulin genes remains to be confirmed.

\section{LJPALI acts during the early stages of nodulation, likely} by regulating the $\mathrm{SA}$-dependent defense pathway.

Rhizobial and pathogenic bacteria have adopted similar strategies and genetic traits to colonize, invade, and establish effective infections in plant hosts (Soto et al. 2006). It has been previously reported that the suppression of $P A L$ expression in plants reduces levels of SA and increased susceptibility to pathogen infection (Huang et al. 2010; Kim and Hwang 2014; Pallas et al 1996). Similarly, both application of exogenous SA and changes in the levels of endogenous SA affect nodule numbers but not Nod factor-induced root hair deformation in legumes (Chen et al. 2014; Martínez-Abarca et al 1998; Stacey et al. 2006; van Spronsen et al. 2003). In our experiments, LjPAL1-OE plants exhibited increased levels of SA accumulation and $P R 1$ expression relative to wild-type plants, were less susceptible to rhizobial infection, and suppressed nodulin gene expression upon M. loti infection (Figs. 5 and 7). These results suggest that $L j P A L 1$ may have an effect on the early stages of rhizobium-legume symbiosis by regulating SA-dependent defense signaling.

In Arabidopsis, treatment with PAL inhibitors completely abolishes resistance to the downy mildew oomycete Hyaloperonospora parasitica. The lost resistance can be fully restored by treatment with exogenous SA (Mauch-Mani and Slusarenko 1996). In our study, the rhizobial infection phenotype of LjPAL1 RNAi plants was partially rescued by exogenous SA treatment (Fig. 10). It is likely that SA biosynthesis is not the only process to which PAL contributes in $L$. japonicus nodulation, because changes in lignin levels regulated by LjPAL1 also play important roles in rhizobial infection. It has been reported that SA levels correlate with the extent of lignin reduction in a series of transgenic alfalfa lines (Lee et al. 2011). Although reductions of both lignin and SA levels were observed in LjPAL1i plants (Figs. 3 and 9), it is not yet clear how endogenous SA interacts with lignin in L. japonicus nodulation.

\section{LjPAL1 plays roles in L. japonicus-pathogen interactions but a negative role in $L$. japonicus-rhizobium symbiosis.}

The transcript levels of plant $P A L$ genes increase following exposure to pathogens and their inducers and there is a positive correlation between plant resistance to pathogens and $P A L$ gene expression (Pellegrini et al. 1994). The downregulation of $P A L$ genes in plants increases their susceptibility to pathogens, while PAL overexpression has the opposite effect (Cass et al. 2015; Kim and Hwang 2014). These results were confirmed in our study. LjPALi plants exhibited reduced resistance to $P$. syringae pv.phaseolicola NPS3121 infection, whereas LjPAL1-OE lines exhibited the opposite response (Fig. 8). It thus appears that the regulation of lignin and endogenous SA levels by the LjPAL1 gene enhances the response to pathogenic infections in L. japonicus (Fig. 11).

As regards the function of the LjPALI gene in L. japonicusrhizobium symbiosis, the elements of phenylpropanoid metabolism that are involved in L. japonicus nodulation can be activated by Me-JA (Figs. 1B and 9B). On the one hand, PAL affects nodule structure and modulates lignin biosynthesis to delay rhizobial infection in LjPAL1-OE lines or accelerates infection in LjPALI $i$ lines. On the other hand, the expression of $L j P A L 1$ was instantaneously activated and subsequently suppressed when MG-20 plants were inoculated with M. loti
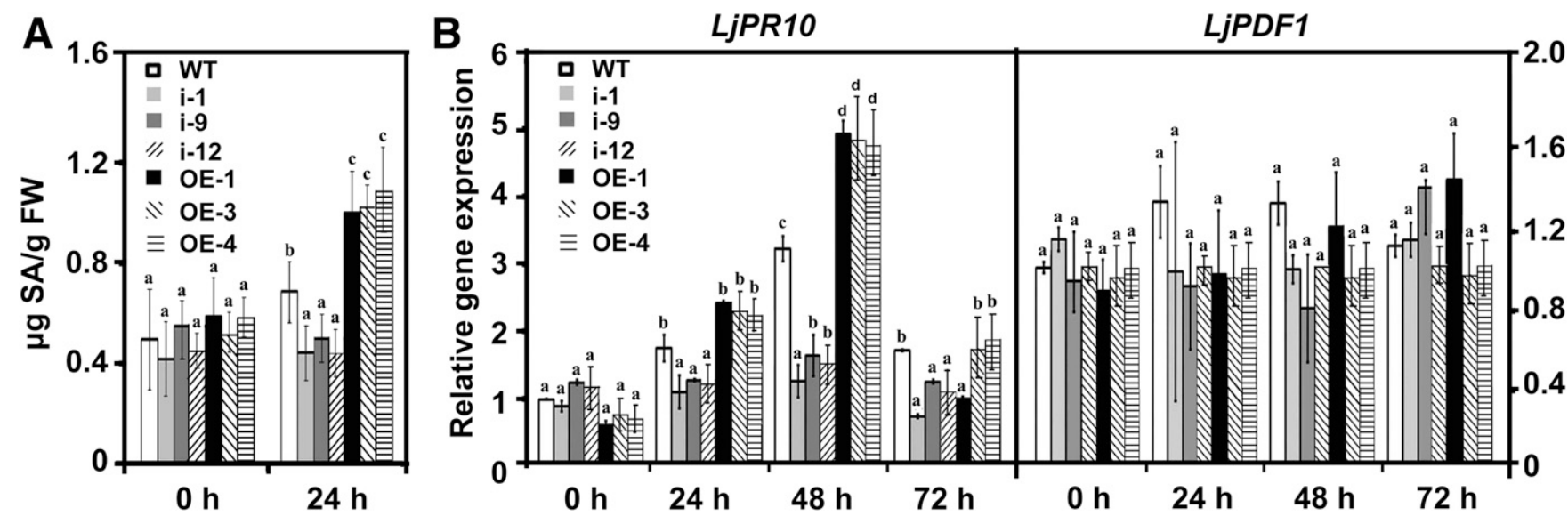

Fig. 9. Endogenous salicylic acid (SA) contents and expression of SA-dependent defense genes in wild-type (WT) and transgenic plant roots following inoculation with Mesorhizobium loti. A, Endogenous SA was measured in M. loti-treated MG-20 and transgenic roots 24 h after treatment. Values are the means of three biological replicates and standard deviations for 8 to 15 independent roots. B, The expression of the SA-dependent marker gene $L j P R 10$ and jasmonic acid-dependent marker gene $L j P D F$ in roots was checked at different time points after $M$. loti treatment, as indicated. The data are averages of triplicate RNA preparations, and error bars indicate the standard errors of the data resulting from three independent experiments. Statistical significances according to Fisher's protected least significant difference test $(P<0.05)$ are indicated by different letters above the data points. 


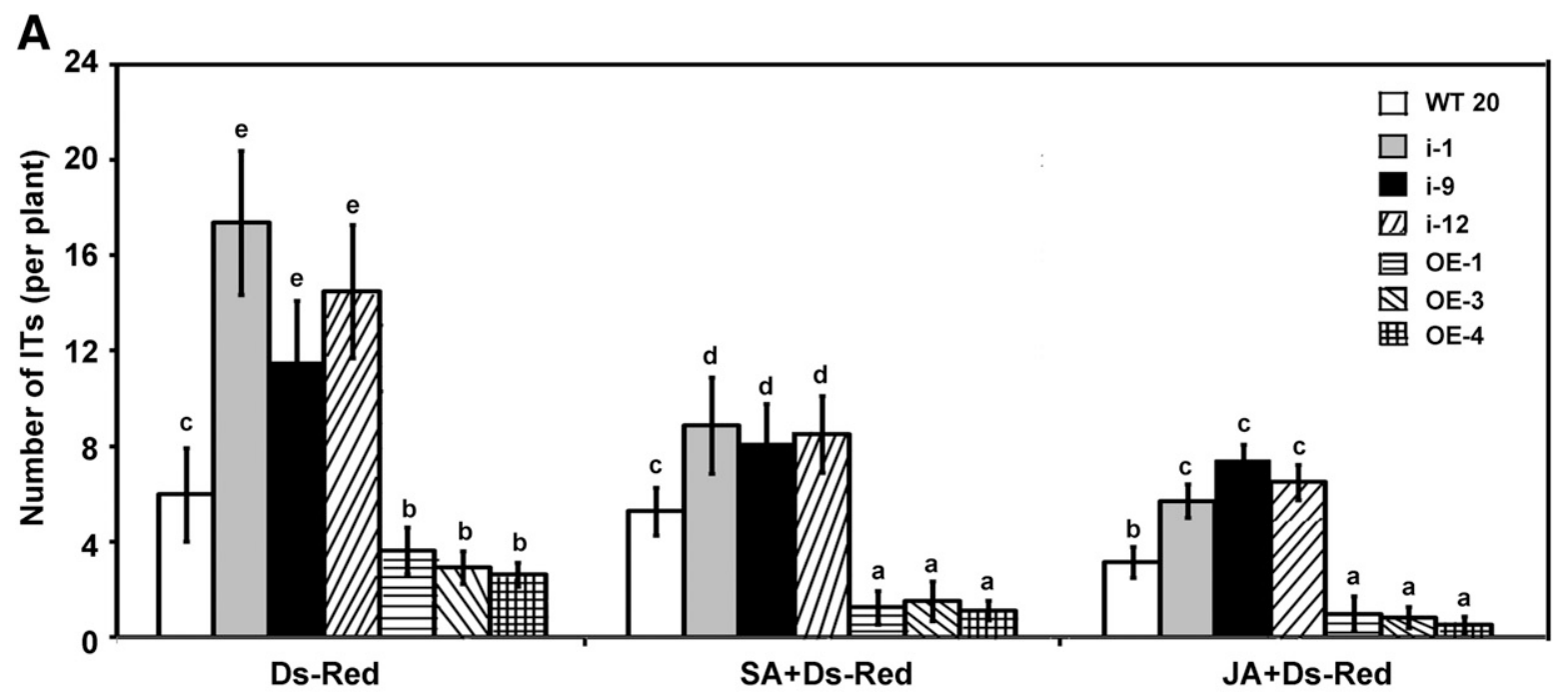

B
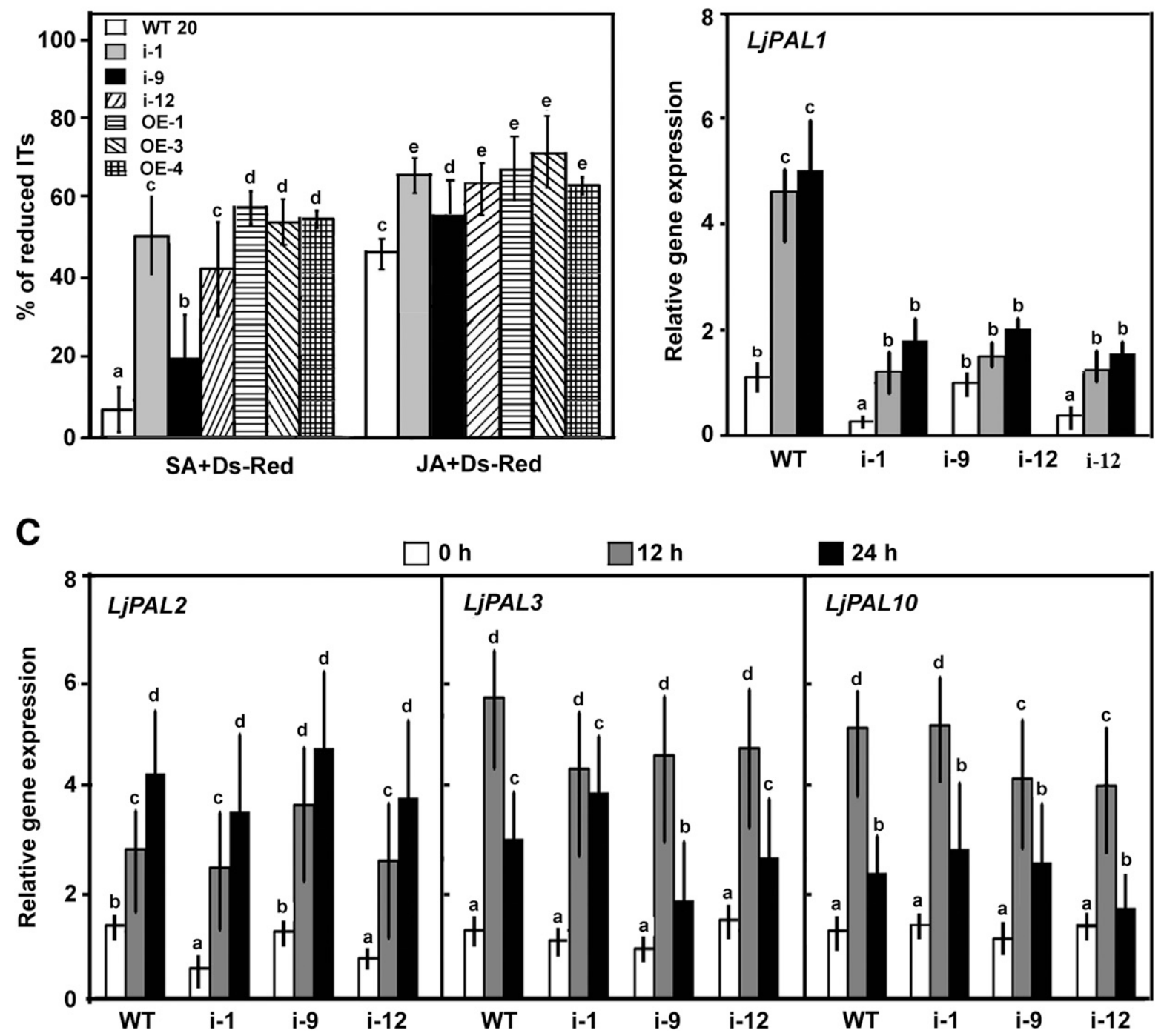

Fig. 10. Exogenous salicylic acid (SA) and methyl-jasmonate (Me-JA) application on MG-20 and LjPAL1 transgenic roots. A, Number of infection threads (ITs) formed on plants grown on agar slants inoculated with Mesorhizobium loti expressing DsRED for 7 days after treatment of the roots with a negative control (blank), $100 \mu \mathrm{M} \mathrm{SA}$, and $100 \mu \mathrm{M}$ jasmonic acid (JA) for $6 \mathrm{~h}$. B, The effect of SA and JA treatment on number of ITs (expressed as a percentage of the number observed in control roots) for wild-type and transgenic plants; $n=40$ to 50 for IT counts. C, Expression of $L j P A L 1$ and its homologous genes in wildtype and $L j P A L 1 i$ roots after treatment with $100 \mu \mathrm{M}$ Me-JA. The data are averages of triplicate RNA preparations, and error bars indicate the standard errors of the data resulting from three independent experiments. Statistical significances according to Fisher's protected least significant difference test $(P<0.05)$ are indicated by different letters above the data points. 
(Fig. 1D). Moreover, rhizobial infection is accelerated in $L j P A L i$ plants but slowed down in LjPAL1-OE plants (Fig. 5). Therefore, PAL has an effect on the early stages of the rhizobiumL. japonicus interaction and regulates endogenous SA biosynthesis as well as the associated defense signaling (Fig. 11).

\section{MATERIALS AND METHODS}

\section{Inoculation with rhizobia and pathogens.}

The MAFF303099 strain of $M$. lot $i$ was diluted to an inoculum optical density at $600 \mathrm{~nm}$ of 0.01 . IT counting was performed using the $M$. loti MAFF303099 strain, which carries a chromosomal DsRed insertion.

The bacterial pathogens $P$. syringae pv. tomato DC3000 and Pseudomonas syringae pv.phaseolicola NPS3121 were grown in King's B broth (per liter, $10 \mathrm{~g}$ of peptone, $1.5 \mathrm{~g}$ of $\mathrm{K}_{2} \mathrm{HPO}_{4}$, $15 \mathrm{~g}$ of glycerol, and $5 \mathrm{~g}$ of $\mathrm{MgSO}_{4}$ ). Roots of L. japonicus plants were infiltrated with bacterial strains in $10 \mathrm{mM} \mathrm{MgCl}_{2}$, using a syringe without a needle. Plants were monitored daily over a 5- to 14-day period for symptom development and bacterial multiplication. Necrotic speck lesions produced by the bacterial pathogen were observed.

\section{Plant growth condition and nodulation assays.}

All L. japonicus lines used in this study were of the MG-20 ecotype. Seeds were scarified for $5 \mathrm{~min}$ in $\mathrm{H}_{2} \mathrm{SO}_{4}$ and were sterilized with $2 \%$ sodium hypochlorite for $10 \mathrm{~min}$. For nodulation assays and IT counts, 3-day-old seedlings were transferred to vertical plates, with filter paper, on $1 \%$ agar containing $1 / 4$ Broughton and Dilworth (B\&D) nutrients in the presence or absence of $\mathrm{KNO}_{3}$, together with $100 \mu \mathrm{M}$ SA or $100 \mu \mathrm{M}$ Me-JA, if required for the experiment at hand. ITs were counted at the indicated times after inoculation by placing whole roots on microscope slides to allow counting of the root surface. For phenotyping in open pots, 3-day-old seedlings were transferred to pots containing vermiculite watered with nitrate-free $1 / 2$ B\&D.

\section{Plasmid constructs and plant transformation.}

The LjPAL1 promoter- $\beta$-glucuronidase (GUS) fusion construct was prepared by PCR amplification of a 1,800-bp sequence upstream of the ATG start codons of LjPAL1, using genomic DNA as the template and the primers specified in Supplementary Table S2. The resulting PCR products were cloned into the binary vector pCAMBIA1391Z, using InFusion PCR cloning kits.

For the RNAi construct, a 140-bp cDNA fragment of $L j P A L 1$ containing a box that is not conserved in other $L j P A L$ genes was amplified by PCR from LjPAL1 cDNA. The amplification products were digested with $S a c \mathrm{I} / \mathrm{Bam} \mathrm{HI}$ and $S a l \mathrm{I} / \mathrm{KpnI}$ and were ligated into the pBS-in vector (Chen et al. 2014). The resulting hairpin structures were cloned into the pCAMBIA1301 vector under the control of the Cauliflower mosaic virus (CaMV) 35S promoter and RBCS terminator.

To generate a construct that would induce $L j P A L 1$ overexpression in plants, the full-length $L j P A L 1$ cDNA was cloned into the pCAMBIA1301 vector under the control of the CaMV $35 \mathrm{~S}$ promoter using In-Fusion PCR cloning kits.

L. japonicus was transformed using a previously described Agrobacterium-mediated transformation procedure (Chen et al. 2014). Hypocotyls excised from $L$. japonicus MG-20 seedlings were infected with Agrobacterium tumefaciens AGL1 harboring the $L j P A L 1$ constructs. Regenerated plants showing hygromycin resistance were then grown in vermiculite pots to harvest the $T_{1}$ seeds. Homozygous seeds of $T_{1}$ transgenic plants were used for further experimentation.

\section{RNA isolation and expression analysis.}

Total RNA was extracted from tissues of L. japonicus, using the RNeasy plant mini kit (Qiagen) following the manufacturer's instructions, and the isolated RNA was treated with RNase-free DNase I (Roche). First-strand cDNA was synthesized from $2 \mu \mathrm{g}$ of total RNA, using M-MLV reverse transcription (Promega) according to the manufacturer's instructions. Real time RT-PCR was performed on a LightCycler 480 instrument. Forty cycles were performed, using the following cycling conditions: $95^{\circ} \mathrm{C}$ for $30 \mathrm{~s}, 95^{\circ} \mathrm{C}$ for $5 \mathrm{~s}, 60^{\circ} \mathrm{C}$ for $20 \mathrm{~s}$, and $72^{\circ} \mathrm{C}$ for $20 \mathrm{~s}$. A Lightcycler480 instrument and Lightcycler480 SYBR green I master (Roche Diagnostics GmbH) were used for real time quantitative PCR amplification; ATPsynthase, Ubiquitin-conjugating enzyme, and Protein phosphatase $2 A$ were used as reference genes (Czechowski et al. 2005). Relative quantification software (Roche) was used to calculate normalized efficiency-corrected relative transcript levels.

\section{Histochemical staining and microscopic analysis.}

Staining with GUS was performed as described previously (Chen et al. 2014). Plants were submerged in GUS reaction buffer (1 mM X-Gluc, 0.1 M phosphate buffer, pH 7.0, $1 \mathrm{mM}$ EDTA, pH 8.0, $0.5 \mathrm{mM} \mathrm{K}_{3}\left[\mathrm{Fe}(\mathrm{CN})_{6}\right], 0.5 \mathrm{mM} \mathrm{K}$ [ $\left.\mathrm{Fe}(\mathrm{CN})_{6}\right]$, $20 \%$ methanol, $0.1 \%$ Triton $\mathrm{X}-100$ ), were infiltrated in vacuum for 20 to $30 \mathrm{~min}$, and then, were maintained at $37^{\circ} \mathrm{C}$ for 4 to $6 \mathrm{~h}$. After staining, nodules from transgenic and wild-type plants were fixed in $2.5 \%$ glutaraldehyde in $0.1 \mathrm{M}$ sodium cacodylate $(\mathrm{pH} 7.0)$ overnight at $4^{\circ} \mathrm{C}$. The fixed nodules were dehydrated by two washes in distilled water and three washes in 2, 2dimethoxypropane solution before transversal sectioning. The resulting thin sections $(4 \mathrm{~mm})$ were observed under a light microscope.

For electron microscopy, different tissues from transgenic and wild-type plants at 2 weeks after inoculation were fixed in $2.5 \%$ glutaraldehyde in $0.1 \mathrm{M}$ sodium phosphate buffer $(\mathrm{pH}$ 7.4) overnight at $4{ }^{\circ} \mathrm{C}$. The fixed tissues were dehydrated by means of sequential washes with $30,50,70,80,90$, and $100 \%$ ethanol, followed by three washes in xylenes, and finally, embedded in wax for longitudinal and transversal sectioning. Thin sections (6 to $8 \mathrm{~mm}$ ) were stained with toluidine blue for light microscopy.

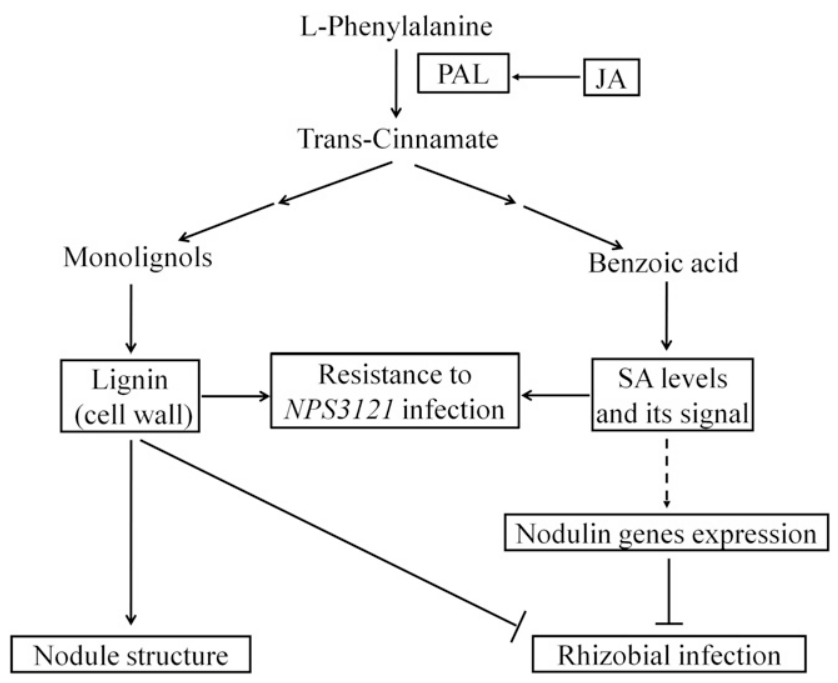

Fig. 11. Speculative working model of the functional role of phenylalanine ammonia lyase in Lotus japonicus-rhizobium symbiosis and L. japonicuspathogen interactions. 
For semithickness sectioning and TEM, samples were fixed in $2.5 \%$ glutaraldehyde for up to $24 \mathrm{~h}$, were washed with $0.1 \mathrm{M}$ sodium phosphate buffer $(\mathrm{pH} 7.2)$ and $1 \%$ osmic acid, were dehydrated by means of sequential washes with 30, 50, 70, 80, 90 , and $100 \%$ ethanol, were immersed in a mixture of epoxy propane and EP812, and finally, were embedded in resin. Semithickness and ultrathin sections were then cut from the resin-embedded products.

\section{PAL activity assay.}

Fresh roots or shoots (usually 0.2 to $0.5 \mathrm{~g}$ ) were ground in an ice-cooled mortar and pestle. The resulting powder was then extracted with $5 \mathrm{ml} 50 \mathrm{mM}$ borate buffer ( $\mathrm{pH} \mathrm{8.8)} \mathrm{containing}$ $15 \mathrm{mM} \beta$-mercaptoethanol. The homogenate was filtered and centrifuged at $10,000 \times g$ at $4^{\circ} \mathrm{C}$ for $20 \mathrm{~min}$ and the supernatant was collected. PAL activity was assayed as described previously (Nadernejad et al. 2013), using a reaction system comprising $1 \mathrm{ml}$ of the extraction buffer, $0.5 \mathrm{ml}$ of $10 \mathrm{mM}$ L-phenylalanine, and $1 \mathrm{ml}$ of enzyme extract. This mixture was incubated at $37^{\circ} \mathrm{C}$ for $1 \mathrm{~h}$, after which, the reaction was terminated by adding $0.5 \mathrm{ml}$ of $6 \mathrm{M} \mathrm{HCl}$, and the product was extracted with $10 \mathrm{ml}$ of ethyl acetate followed by evaporation to remove the extracting solvent. The solid residue was suspended in $3 \mathrm{ml}$ of $0.05 \mathrm{M}$ $\mathrm{NaOH}$ and its cinnamic acid content was determined spectrophotometrically by measuring the absorbance of the resulting solution at $290 \mathrm{~nm} ; 1$ unit of PAL activity is equal to $1 \mu \mathrm{mol}$ of cinnamic acid produced per min (Kim and Hwang 2014). Protein levels were estimated spectrophotometrically by comparing the samples' absorption at 280 and $260 \mathrm{~nm}$, using bovine serum albumin as a standard.

\section{Measurement of lignin content.}

Lignin content was determined according to the modified method of Nozaki (2012). Materials stored at $-70^{\circ} \mathrm{C}$ were homogenized in ice-cold $95 \%$ ethanol using a plastic pestle and, then, using an ultrasonic disruptor. The homogenate was centrifuged at $3,000 \times g$ for $5 \mathrm{~min}$. The resulting pellet was washed three times with $95 \%$ ethanol and twice with a 1:2 (vol/vol) mixture of ethanol and hexane and was then allowed to air-dry at $36^{\circ} \mathrm{C}$ overnight. The dried pellet was ultrasonically homogenized in acetic acid and centrifuged at 3,000 $\times g$ for 5 min. The pellet was resuspended in $25 \%$ acetyl bromide in acetic acid and was centrifuged at 3,000 $\times g$ for $5 \mathrm{~min}$. To each sample, $300 \mathrm{ml} 25 \%$ acetyl bromide in acetic acid was added. After heating to $70^{\circ} \mathrm{C}$ for $30 \mathrm{~min}, 270 \mathrm{ml}$ of $2 \mathrm{M} \mathrm{NaOH}, 1.5 \mathrm{ml}$ of acetic acid, $30 \mathrm{ml}$ of $7.5 \mathrm{M}$ hydroxylamine hydrochloride, and $900 \mathrm{ml}$ of acetic acid were added to each sample. The samples were centrifuged at $3,000 \times g$ for $5 \mathrm{~min}$, and the absorbance of the supernatant was measured at $280 \mathrm{~nm}$ to determine the lignin content, using pure lignin as a standard curve (Nozaki 2012).

\section{SA measurement.}

Two-week-old seedlings of the wild-type and LjPAL1 transgenic lines were collected, weighed, and then frozen in liquid nitrogen. Total SA extraction was performed using 500-mg tissue samples, as described in our previous report (Chen et al. 2014). High-performance liquid chromatography analyses were performed on an LC-20AT instrument (Shimadzu), using 20-ml subsamples of the final methanolic plant extracts (which had an initial volume of $200 \mathrm{ml}$ each). The subsamples were injected onto a C18 column ( $25 \mathrm{~cm} \times 4.6 \mathrm{~mm}$; Waters $)$. SA was separated isocratically with $30 \%$ (vol/vol) methanol containing $1 \%$ ( $\mathrm{vol} / \mathrm{vol}$ ) acetic acid, at a flow rate of $1 \mathrm{ml} \mathrm{min}^{-1}$ and an oven temperature of $40^{\circ} \mathrm{C}$. SA concentrations were calculated from absorbencies at $235 \mathrm{~nm}$.

\section{Statistical analysis.}

The results are expressed as means \pm standard errors. Statistical analysis was performed using Student's $t$ test, Fisher's protected least significant difference test to determine significance using Super analysis of variance software (Abacus Concepts).

\section{ACKNOWLEDGMENTS}

We thank J. Stougaard and K. Markmann for M. loti MAFF303099 strain carrying the chromosomal DsRed insertion (Aarhus University); X. Li for bacterial pathogens $P$. syringae pv. tomato DC3000 and P. syringae pv. phaseolicola NPS3121(NPS3121). This work was supported by the National Natural Science Foundation of China (31570242, 31572195), the Natural Science Foundation of Guangdong (2014A030313713), and the Externa1 Cooperation Program of BIC, Chinese Academy of Sciences (151644KYSB20130054).

\section{LITERATURE CITED}

Boerjan, W., Ralph, J., and Baucher, M. 2003. Lignin biosynthesis. Annu. Rev. Plant Biol. 54:519-546.

Carocha, V., Soler, M., Hefer, C., Cassan-Wang, H., Fevereiro, P., Myburg, A. A., Paiva, J. A., and Grima-Pettenati, J. 2015. Genome-wide analysis of the lignin toolbox of Eucalyptus grandis. New Phytol. 206:1297-1313.

Cass, C. L., Peraldi, A., Dowd, P. F., Mottiar, Y., Santoro, N., Karlen, S. D., Bukhman, Y. V., Foster, C. E., Thrower, N., Bruno, L. C., Moskvin, O. V., Johnson, E. T., Willhoit, M. E., Phutane, M., Ralph, J., Mansfield, S. D., Nicholson, P., and Sedbrook, J. C. 2015. Effects of PHENYLALANINE AMMONIA LYASE (PAL) knockdown on cell wall composition, biomass digestibility, and biotic and abiotic stress responses in Brachypodium. J. Exp. Bot. 66:4317-4335.

Chaman, M. E., Copaja, S. V., and Argandoña, V. H. 2003. Relationships between salicylic acid content, phenylalanine ammonia-lyase (PAL) activity, and resistance of barley to aphid infestation. J. Agric. Food Chem. 51:2227-2231.

Chen, W., Li, X., Tian, L., Wu, P., Li, M., Jiang, H., Chen, Y., and Wu, G. 2014. Knockdown of LjALD1, AGD2-like defense response protein 1, influences plant growth and nodulation in Lotus japonicus. J. Integr. Plant Biol. 56:1034-1041.

Cochrane, F. C., Davin, L. B., and Lewis, N. G. 2004. The Arabidopsis phenylalanine ammonia lyase gene family: Kinetic characterization of the four PAL isoforms. Phytochemistry 65:1557-1564.

Czechowski, T., Stitt, M., Altmann, T., Udvardi, M. K., and Scheible, W. R. 2005. Genome-wide identification and testing of superior reference genes for transcript normalization in Arabidopsis. Plant Physiol. 139: 5-17.

de Jong, F., Hanley, S. J., Beale, M. H., and Karp, A. 2015. Characterisation of the willow phenylalanine ammonia-lyase $(P A L)$ gene family reveals expression differences compared with poplar. Phytochemistry 117:90-97.

Deguchi, Y., Banba, M., Shimoda, Y., Chechetka, S. A., Suzuri, R., Okusako, Y., Ooki, Y., Toyokura, K., Suzuki, A., Uchiumi, T., Higashi, S., Abe, M., Kouchi, H., Izui, K., and Hata, S. 2007. Transcriptome profiling of Lotus japonicus roots during arbuscular mycorrhiza development and comparison with that of nodulation. DNA Res. 14: 117-133.

Dixon, R. A., and Paiva, N. L. 1995. Stress-induced phenylpropanoid metabolism. Plant Cell 7:1085-1097.

Dong, C. J., and Shang, Q. M. 2013. Genome-wide characterization of phenylalanine ammonia-lyase gene family in watermelon (Citrullus lanatus). Planta 238:35-49.

Ejtahed, R. S., Radjabian, T., and Hoseini Tafreshi, S. A. 2015. Expression analysis of phenylalanine ammonia lyase gene and rosmarinic acid production in Salvia officinalis and Salvia virgata shoots under salicylic acid elicitation. Appl. Biochem. Biotechnol. 176:1846-1858.

Elkind, Y., Edwards, R., Mavandad, M., Hedrick, S. A., Ribak, O., Dixon, R. A., and Lamb, C. J. 1990. Abnormal plant development and down-regulation of phenylpropanoid biosynthesis in transgenic tobacco containing a heterologous phenylalanine ammonia-lyase gene. Proc. Natl. Acad. Sci. U.S.A. 87: 9057-9061.

Frei, M. 2013. Lignin: Characterization of a multifaceted crop component. Sci. World J. 2013:436517.

Gallego-Giraldo, L., Bhattarai, K., Pislariu, C. I., Nakashima, J., Jikumaru, Y., Kamiya, Y., Udvardi, M. K., Monteros, M. J., and Dixon, R. A. 2014. Lignin modification leads to increased nodule numbers in alfalfa. Plant Physiol. 164:1139-1150. 
Geurts, R., and Bisseling, T. 2002. Rhizobium nod factor perception and signalling. Plant Cell 14 (Suppl):S239-S249.

Huang, J., Gu, M., Lai, Z., Fan, B., Shi, K., Zhou, Y. H., Yu, J. Q., and Chen, Z. 2010. Functional analysis of the Arabidopsis $P A L$ gene family in plant growth, development, and response to environmental stress. Plant Physiol. 153:1526-1538.

Jones, J. D. G., and Dangl, J. L. 2006. The plant immune system. Nature 444:323-329.

Kim, D. S., and Hwang, B. K. 2014. An important role of the pepper phenylalanine ammonia-lyase gene (PAL1) in salicylic acid-dependent signalling of the defence response to microbial pathogens. J. Exp. Bot. 65:2295-2306.

Kouchi, H., Shimomura, K., Hata, S., Hirota, A., Wu, G. J., Kumagai, H., Tajima, S., Suganuma, N., Suzuki, A., Aoki, T., Hayashi, M., Yokoyama, T., Ohyama, T., Asamizu, E., Kuwata, C., Shibata, D., and Tabata, S 2004. Large-scale analysis of gene expression profiles during early stages of root nodule formation in a model legume, Lotus japonicus. DNA Res. 11:263-274.

Lee, Y., Chen, F., Gallego-Giraldo, L., Dixon, R. A., and Voit, E. O. 2011. Integrative analysis of transgenic alfalfa (Medicago sativa L.) suggests new metabolic control mechanisms for monolignol biosynthesis. PLOS Comput. Biol. 7:e1002047.

Liang, Y., Cao, Y., Tanaka, K., Thibivilliers, S., Wan, J., Choi, J., Kang, Ch., Qiu, J., and Stacey, G. 2013. Nonlegumes respond to rhizobial Nod factors by suppressing the innate immune response. Science 341:1384-1387.

Lohar, D. P., Sharopova, N., Endre, G., Peñuela, S., Samac, D., Town, C., Silverstein, K. A. T., and VandenBosch, K. A. 2006. Transcript analysis of early nodulation events in Medicago truncatula. Plant Physiol. 140:221-234.

Lopez-Gomez, M., Sandal, N., Stougaard, J., and Boller, T. 2012. Interplay of flg22-induced defence responses and nodulation in Lotus japonicus. J. Exp. Bot. 63:393-401.

MacDonald, M. J., and D'Cunha, G. B. 2007. A modern view of phenylalanine ammonia lyase. Biochem. Cell Biol. 85:273-282.

Madsen, L. H., Tirichine, L., Jurkiewicz, A., Sullivan, J. T., Heckmann, A. B., Bek, A. S., Ronson, C. W., James, E. K., and Stougaard, J. 2010. The molecular network governing nodule organogenesis and infection in the model legume Lotus japonicus. Nat. Commun. 1:10.

Martínez-Abarca, F., Herrera-Cervera, J. A., Bueno, P., Sanjuan, J., Bisseling, T., and Olivares, J. 1998. Involvement of salicylic acid in the establishment of the Rhizobium meliloti-alfalfa symbiosis. Mol. Plant Microbe Interact. 11:153-155.

Mauch-Mani, B., and Slusarenko, A. J. 1996. Production of salicylic acid precursors is a major function of phenylalanine ammonia-lyase in the resistance of Arabidopsis to Peronospora parasitica. Plant Cell 8:203-212.

Nadernejad, N., Ahmadimoghadam, A., Hossyinifard, J., and Poorseyed,i S. 2013. Effect of different rootstocks on PAL activity and phenolic compounds in flowers, leaves, hulls and kernels of three pistachio (Pistacia vera L.) cultivars. Trees 27:1681-1689.

Nozaki, M., Sugiyama, M., Duan, J., Uematsu, H., Genda, T., and Sato, Y. 2012. A missense mutation in the glucosamine-6-phosphate $\mathrm{N}$-acetyltransferase-encoding gene causes temperature-dependent growth defects and ectopic lignin deposition in Arabidopsis. Plant Cell 24 3366-3379.

Nugroho, L. H., Verberne, M. C., and Verpoorte, R. 2002. Activities of enzymes involved in the phenylpropanoid pathway in constitutively salicylic acid-producing tobacco plants. Plant Physiol. Biochem. 40: 755-760.

Pallas, J. A., Paiva, N. L., Lamb, C., and Dixon, R. A. 1996. Tobacco plants epigenetically suppressed in phenylalanine ammonia-lyase expression do not develop systemic acquired resistance in response to infection by tobacco mosaic virus. Plant J. 10:281-293.

Pauwels, L., Morreel, K., De Witte, E., Lammertyn, F., Van Montagu, M., Boerjan, W., Inzé, D., and Goossens, A. 2008. Mapping methyl jasmonate-mediated transcriptional reprogramming of metabolism and cell cycle progression in cultured Arabidopsis cells. Proc. Natl. Acad. Sci. U.S.A. 105:1380-1385.
Payyavula, R. S., Navarre, D. A., Kuhl, J. C., Pantoja, A., and Pillai, S. S. 2012. Differential effects of environment on potato phenylpropanoid and carotenoid expression. BMC Plant Biol. 12:39.

Peleg-Grossman, S., Golani, Y., Kaye, Y., Melamed-Book, N., and Levine, A. 2009. NPR1 protein regulates pathogenic and symbiotic interactions between Rhizobium and legumes and non-legumes. PLoS One 4:e8399.

Pellegrini, L., Rohfritsch, O., Fritig, B., and Legrand, M. 1994. Phenylalanine ammonia-lyase in tobacco. Molecular cloning and gene expression during the hypersensitive reaction to tobacco mosaic virus and the response to a fungal elicitor. Plant Physiol. 106:877-886.

Raes, J., Rohde, A., Christensen, J. H., Van de Peer, Y., and Boerjan, W. 2003. Genome-wide characterization of the lignification toolbox in Arabidopsis. Plant Physiol. 133:1051-1071.

Reichert, A. I., He, X. Z., and Dixon, R. A. 2009. Phenylalanine ammonialyase (PAL) from tobacco (Nicotiana tabacum): Characterization of the four tobacco $P A L$ genes and active heterotetrameric enzymes. Biochem. J. 424:233-242.

Rich, M. K., Schorderet, M., and Reinhardt, D. 2014. The role of the cell wall compartment in mutualistic symbioses of plants. Front. Plant Sci. $5: 238$.

Rohde, A., Morreel, K., Ralph, J., Goeminne, G., Hostyn, V., De Rycke, R., Kushnir, S., Van Doorsselaere, J., Joseleau, J. P., Vuylsteke, M., Van Driessche, G., Van Beeumen, J., Messens, E., and Boerjan, W. 2004 Molecular phenotyping of the pall and pal2 mutants of Arabidopsis thaliana reveals far-reaching consequences on phenylpropanoid, amino acid, and carbohydrate metabolism. Plant Cell 16:2749-2771.

Soto, M. J., Sanjuán, J., and Olivares, J. 2006. Rhizobia and plantpathogenic bacteria: Common infection weapons. Microbiology 152 3167-3174.

Stacey, G., McAlvin, C. B., Kim, S. Y., Olivares, J., and Soto, M. J. 2006. Effects of endogenous salicylic acid on nodulation in the model legumes Lotus japonicus and Medicago truncatula. Plant Physiol. 141:1473-1481.

Taheri, P., and Tarighi, S. 2010. Riboflavin induces resistance in rice against Rhizoctonia solani via jasmonate-mediated priming of phenylpropanoid pathway. J. Plant Physiol. 167:201-208.

Takeda, N., Okamoto, S., Hayashi, M., and Murooka, Y. 2005. Expression of $L j E N O D 40$ genes in response to symbiotic and non-symbiotic signals: LjENOD40-1 and LjENOD40-2 are differentially regulated in Lotus japonicus. Plant Cell Physiol. 46:1291-1298.

Tsai, C. J., Harding, S. A., Tschaplinski, T. J., Lindroth, R. L., and Yuan, Y. 2006. Genome-wide analysis of the structural genes regulating defense phenylpropanoid metabolism in Populus. New Phytol. 172:47-62.

van Spronsen, P. C., Tak, T., Rood, A. M., van Brussel, A. A., Kijne, J. W., and Boot, K. J. 2003. Salicylic acid inhibits indeterminate-type nodulation but not determinate-type nodulation. Mol. Plant-Microbe Interact 16:83-91.

Wanner, L. A., Li, G., Ware, D., Somssich, I. E., and Davis, K. R. 1995. The phenylalanine ammonia-lyase gene family in Arabidopsis thaliana. Plant Mol. Biol. 27:327-338.

Wu, Z., Gui, S., Wang, S., and Ding, Y. 2014. Molecular evolution and functional characterisation of an ancient phenylalanine ammonia-lyase gene (NnPAL1) from Nelumbo nucifera: Novel insight into the evolution of the PAL family in angiosperms. BMC Evol. Biol. 14:100.

Yang, S., Tang, F., Gao, M., Krishnan, H. B., and Zhu, H. 2010. $R$ gene-controlled host specificity in the legume-rhizobia symbiosis. Proc. Natl. Acad. Sci. U.S.A. 107:18735-18740

Yoro, E., Suzaki, T., Toyokura, K., Miyazawa, H., Fukaki, H., and Kawaguchi, M. 2014. A positive regulator of nodule organogenesis, NODULE INCEPTION, acts as a negative regulator of Rhizobial infection in Lotus japonicus. Plant Physiol. 165:747-758.

\section{AUTHOR-RECOMMENDED INTERNET RESOURCES}

The Gene Index Project Phytozome database: https://phytozome.jgi.doe.gov

The miyakogusa.jp 3.0 database: http://www.kazusa.or.jp/lotus/index.html 